\title{
Parametric geometry of numbers and applications
}

\author{
by \\ WolfGang M. Schmidt (Boulder, CO) and \\ LeOnHARD Summerer (Wien)
}

1. Introduction. A basic result in the Geometry of Numbers is Minkowski's Second Convex Body Theorem. Given a closed symmetric convex body $K$ in $\mathbb{R}^{n}$ and a lattice $\Lambda$ in $\mathbb{R}^{n}$, the $i$ th successive minimum $\lambda_{i}, 1 \leq$ $i \leq n$, with respect to $K$ and $\Lambda$ is the least number $\lambda>0$ for which $\lambda K$ contains $i$ linearly independent lattice points. Clearly, $\lambda_{1} \leq \cdots \leq \lambda_{n}$ and Minkowski's Theorem ([8]) says that

$$
\frac{2^{n}}{n !} \frac{\operatorname{det} \Lambda}{\operatorname{Vol}(K)} \leq \lambda_{1} \cdots \lambda_{n} \leq 2^{n} \frac{\operatorname{det} \Lambda}{\operatorname{Vol}(K)},
$$

where $\operatorname{Vol}(K)$ is the volume of $K$ and $\operatorname{det} \Lambda$ the determinant of $\Lambda$.

Suppose $\mu_{1}, \ldots, \mu_{n}$ are reals with $\mu_{1}+\cdots+\mu_{n}=0$, and for $Q>1$ let $T_{Q}: \mathbb{R}^{n} \rightarrow \mathbb{R}^{n}$ be the linear map with

$$
\mathbf{p}:=\left(p_{1}, \ldots, p_{n}\right) \mapsto\left(Q^{\mu_{1}} p_{1}, \ldots, Q^{\mu_{n}} p_{n}\right) .
$$

Then a symmetric convex body $K$ gives rise to the bodies $K(Q):=T_{Q}(K)$ parametrized by $Q$. We propose to study the successive minima $\lambda_{1}(Q)$, $\ldots, \lambda_{n}(Q)$ with respect to $K(Q), \Lambda$ as functions of $Q$. Trivially,

$$
0<\lambda_{1}(Q) \leq \cdots \leq \lambda_{n}(Q),
$$

and since $\operatorname{Vol}(K(Q))=\operatorname{Vol}(K)$, Minkowski's Theorem gives

$$
c_{1}(K, \Lambda) \leq \lambda_{1}(Q) \cdots \lambda_{n}(Q) \leq c_{2}(K, \Lambda),
$$

where $c_{1}(K, \Lambda), c_{2}(K, \Lambda)$ depend only on $K, \Lambda$.

Our study is inspired by Diophantine Approximation where, beginning with Dirichlet's Theorem, a family of systems of inequalities parametrized by $Q>1$ comes into play. Suppose $n>1$,

$$
\mu_{1}=1, \quad \mu_{2}=\cdots=\mu_{n}=-1 /(n-1),
$$

2010 Mathematics Subject Classification: 11H06, 11J13.

Key words and phrases: lattice, successive minima, simultaneous approximation, approximation constants. 
and let $\xi_{1}, \ldots, \xi_{n-1}$ be real numbers. Then Dirichlet's Theorem on simultaneous approximation asserts that for any $Q>1$ the system of inequalities

$$
\begin{aligned}
|x| & \leq Q^{\mu_{1}}, \\
\left|\xi_{1} x-y_{1}\right| & \leq Q^{\mu_{2}}, \\
& \vdots \\
\left|\xi_{n-1} x-y_{n-1}\right| & \leq Q^{\mu_{n}},
\end{aligned}
$$

has a nontrivial solution in integer points $\mathbf{x}=\left(x, y_{1}, \ldots, y_{n-1}\right)$. When $\mathcal{C}$ is the unit cube of points $\mathbf{p}$ with $\left|p_{i}\right| \leq 1,1 \leq i \leq n$, and $\Lambda=\Lambda(\xi)$ the lattice of points $\mathbf{p}=\left(x, \xi_{1} x-y_{1}, \ldots, \xi_{n-1} x-y_{n-1}\right)$ with $\mathbf{x} \in \mathbb{Z}^{n}$, Dirichlet's Theorem asserts that there is a nonzero lattice point in $\mathcal{C}(Q)$, i.e. that the first minimum $\lambda_{1}(Q)$ with respect to $\mathcal{C}(Q)$ and $\Lambda$ is at most 1 .

Much work in Diophantine Approximation is implicitly about the function $\lambda_{1}(Q)$ and we believe that a study of the complete set of functions $\lambda_{1}(Q), \ldots, \lambda_{n}(Q)$ will lead to new insights.

Suppose now that

$$
\mu_{1}=-1, \quad \mu_{2}=\cdots=\mu_{n}=1 /(n-1),
$$

and let $\Lambda^{*}(\xi)$ be the dual lattice to $\Lambda(\xi)$, which consists of points $\mathbf{p}=$ $\left(x-\xi_{1} y_{1}-\cdots-\xi_{n-1} y_{n-1}, y_{1}, \ldots, y_{n-1}\right)$ with $\mathbf{x} \in \mathbb{Z}^{n}$. Dirichlet's Theorem on linear forms may be interpreted as saying that for any $Q>1$, the body $\mathcal{C}(Q)$ contains a nonzero point of $\Lambda^{*}(\xi)$. Thus if $\nu_{i}(Q), 1 \leq i \leq n$, are the successive minima with respect to $\mathcal{C}(Q)$ and $\Lambda^{*}(\xi)$, then $\nu_{1}(Q) \leq 1$. Again we will be interested in all the functions $\nu_{1}(Q), \ldots, \nu_{n}(Q)$. In the special situation where $\xi_{i}=a^{i}, a \in \mathbb{R} \backslash\{0\}$, see also [7].

The reciprocal body $\mathcal{C}^{*}$ of $\mathcal{C}$ consists of the points $\mathbf{p}$ with $\left|p_{1}\right|+\cdots+\left|p_{n}\right|$ $\leq 1$. Therefore $\mathcal{C}^{*} \subseteq \mathcal{C} \subseteq n \mathcal{C}^{*}$ and the successive minima $\lambda_{i}^{*}(Q)$ of $\mathcal{C}^{*}(Q)$ with respect to $\Lambda^{*}(\xi)$ have

$$
\nu_{i}(Q) \leq \lambda_{i}^{*}(Q) \leq n \nu_{i}(Q) .
$$

In the general context formulated at the beginning, we observe that each $\lambda_{i}(Q)$ is a continuous function of $Q$ since $K(Q)$ is closed. In the next step, we wonder whether for given $s, 1 \leq s<n$, there are arbitrarily large values of $Q$ with

$$
\lambda_{s}(Q)=\lambda_{s+1}(Q) .
$$

When $A=\left\{i_{1}<\cdots<i_{s}\right\} \subseteq\{1, \ldots, n\}$, set $\mu_{A}=\sum_{i \in A} \mu_{i}$ and let $\pi_{A}$ : $\mathbb{R}^{n} \rightarrow \mathbb{R}^{s}$ be the map with

$$
\pi_{A}(\mathbf{p})=\left(p_{i_{1}}, \ldots, p_{i_{s}}\right) \in \mathbb{R}^{s} .
$$


Our result here is as follows:

TheOREM 1.1. Suppose for every s-dimensional space $S$ spanned by lattice points (i.e. points of $\Lambda$ ), there is some $A$ of cardinality $s$ with $\mu_{A}<0$ and $\pi_{A}(S)=\mathbb{R}^{s}$. Then there are arbitrarily large values of $Q$ with $\lambda_{s}(Q)=$ $\lambda_{s+1}(Q)$.

It will be seen in Section 2 that when $1, \xi_{1}, \ldots, \xi_{n-1}$ are linearly independent over $\mathbb{Q}$, Theorem 1.1 applies for each $s, 1 \leq s<n$, in the context of Dirichlet's Theorem on simultaneous approximation, as well as on linear forms.

Thus there are arbitrarily large values of $Q$ with $\lambda_{s}(Q)=\lambda_{s+1}(Q)$ as well as arbitrarily large values of $Q$ with $\nu_{s}(Q)=\nu_{s+1}(Q)$. Also, there are arbitrarily large $Q$ with $\lambda_{s}^{*}(Q)=\lambda_{s+1}^{*}(Q)$.

In general, there is a nonzero lattice point $\mathbf{p}$ in $\lambda_{1}(Q) K(Q)$. This point has

$$
|\mathbf{p}| \geq c_{3}>0 \quad \text { and } \quad|\mathbf{p}| \leq c_{4} \lambda_{1}(Q) Q^{\mu},
$$

where $\mu=\max \left(\mu_{1}, \ldots, \mu_{n}\right)$, so that

$$
\lambda_{1}(Q) \geq c_{5} Q^{-\mu}>0,
$$

and hence by Minkowski's Theorem,

$$
\lambda_{n}(Q) \leq c_{6} Q^{\mu(n-1)} .
$$

Next we define $\psi_{i}(Q)$ for $Q>1$ by

$$
\lambda_{i}(Q)=Q^{\psi_{i}(Q)}, \quad i=1, \ldots, n .
$$

The $\psi_{i}(Q)$ are again continuous and we have $0<\psi_{1}(Q) \leq \cdots \leq \psi_{n}(Q)$, as well as

$$
\left|\psi_{1}(Q)+\cdots+\psi_{n}(Q)\right| \leq c_{7}(K, \Lambda) / \log Q
$$

by Minkowski's Theorem.

The quantities

$$
\bar{\psi}_{i}=\limsup _{Q \rightarrow \infty} \psi_{i}(Q) \quad \text { and } \quad \underline{\psi}_{i}=\liminf _{Q \rightarrow \infty} \psi_{i}(Q)
$$

are finite by $(1.2),(1.3)$ and satisfy the inequalities

$$
\bar{\psi}_{1} \leq \cdots \leq \bar{\psi}_{n} \quad \text { and } \quad \underline{\psi}_{1} \leq \cdots \leq \underline{\psi}_{n}
$$

and also $\bar{\psi}_{i} \geq \underline{\psi}_{i}$ for $i=1, \ldots, n$.

By definition, if $\eta>\bar{\psi}_{s}$ and $Q$ is large we will have $\psi_{s}(Q)<\eta$. Given that there are arbitrarily large values of $Q$ with $\lambda_{s}(Q)=\lambda_{s+1}(Q)$, there will be arbitrarily large values of $Q$ with $\psi_{s+1}(Q)=\psi_{s}(Q)<\eta$ and therefore

$$
\underline{\psi}_{s+1} \leq \bar{\psi}_{s} \text {. }
$$


TheOREM 1.2. For $1 \leq i \leq n$ we have

$$
\begin{aligned}
& \bar{\psi}_{1}+\cdots+\bar{\psi}_{i-1}+\underline{\psi}_{i}+\bar{\psi}_{i+1}+\cdots+\bar{\psi}_{n} \geq 0, \\
& \underline{\psi}_{1}+\cdots+\underline{\psi}_{i-1}+\bar{\psi}_{i}+\underline{\psi}_{i+1}+\cdots+\underline{\psi}_{n} \leq 0 .
\end{aligned}
$$

Suppose now that we are in the context of Dirichlet's Theorem on simultaneous approximation, so that $\Lambda=\Lambda(\xi)$ with $1, \xi_{1}, \ldots, \xi_{n-1}$ linearly independent over $\mathbb{Q}$, and $K=\mathcal{C}$. In this context we have

THEOREM 1.3 .

$$
\begin{aligned}
n \underline{\psi}_{2} & \leq(n-1) \bar{\psi}_{2}+\underline{\psi}_{1}, \\
n \bar{\psi}_{n-1} & \geq(n-1) \underline{\psi}_{n-1}+\bar{\psi}_{n} .
\end{aligned}
$$

We will connect $\bar{\psi}_{1}, \underline{\psi}_{1}$ and $\bar{\psi}_{n}, \underline{\psi}_{n}$ to classical approximation exponents as studied by Khinchin [4], [5], Jarník [3], etc., and most recently by Roy [11] and Bugeaud and Laurent [1], [2].

Given $\xi=\left(\xi_{1}, \ldots, \xi_{n-1}\right)$ with $1, \xi_{1}, \ldots, \xi_{n-1}$ linearly independent over $\mathbb{Q}$, the quantities $\omega$ (resp. $\hat{\omega}$ ) are defined as the supremum of the numbers $\eta$ such that there are arbitrarily large values of $X$ for which (resp. such that for every large value of $X$ ) the system of inequalities

$$
|x| \leq X, \quad\left|\xi_{i} x-y_{i}\right| \leq X^{-\eta} \quad \text { for } i=1, \ldots, n-1,
$$

has a nontrivial solution $\mathbf{x}=\left(x, y_{1}, \ldots, y_{n-1}\right) \in \mathbb{Z}^{n}$.

On the other hand, $\omega^{*}$ (resp. $\hat{\omega}^{*}$ ) is the supremum of the numbers $\eta$ such that there are arbitrarily large values of $X$ for which (resp. such that for every large value of $X$ ) the system

$$
\left|x-\sum_{i=1}^{n-1} \xi_{i} y_{i}\right| \leq X^{-\eta}, \quad\left|y_{i}\right| \leq X \quad \text { for } i=1, \ldots, n-1,
$$

has a nontrivial solution in integer $n$-tuples $\mathbf{x}$. It will not be hard to prove

THEOREM 1.4 .

$$
\begin{aligned}
(\omega+1)\left(1+\underline{\psi}_{1}\right) & =(\hat{\omega}+1)\left(1+\bar{\psi}_{1}\right)=\frac{n}{n-1} \\
\left(\omega^{*}+1\right)\left(\frac{1}{n-1}-\bar{\psi}_{n}\right) & =\left(\hat{\omega}^{*}+1\right)\left(\frac{1}{n-1}-\underline{\psi}_{n}\right)=\frac{n}{n-1} .
\end{aligned}
$$

Consequently, $\omega, \hat{\omega}, \omega^{*}, \hat{\omega}^{*}$ determine $\bar{\psi}_{1}, \underline{\psi}_{1}, \bar{\psi}_{n}, \underline{\psi}_{n}$ and vice versa. We will show that Khinchin's transference principle between $\omega$ and $\omega^{*}$ is equivalent to

$$
\underline{\psi}_{1}+(n-1) \bar{\psi}_{n} \geq 0 \quad \text { and } \quad \bar{\psi}_{n}+(n-1) \underline{\psi}_{1} \leq 0,
$$

which however is weaker than the linear inequalities of Theorem 1.2. 
In the context of Dirichlet's Theorem on simultaneous approximation we now specialize further to the case of dimension two in Jarník's and Laurent's papers, which corresponds to $n=3$ in our notation.

TheOREM 1.5. When $n=3$, then

$$
\bar{\psi}_{1}+\underline{\psi}_{3}+2 \bar{\psi}_{1} \underline{\psi}_{3}=0 \text {. }
$$

Theorem 1.6. When $n=3$, then also

$$
\begin{aligned}
& 2 \underline{\psi}_{1}+\bar{\psi}_{3} \leq-\underline{\psi}_{3}\left(3+2 \underline{\psi}_{1}+4 \bar{\psi}_{3}\right), \\
& 2 \bar{\psi}_{3}+\underline{\psi}_{1} \geq-\bar{\psi}_{1}\left(3+2 \bar{\psi}_{3}+4 \underline{\psi}_{1}\right) .
\end{aligned}
$$

On account of (1.11) the relations (1.12), (1.13) are equivalent to

$$
\begin{aligned}
& 2 \underline{\psi}_{1}+\bar{\psi}_{3} \leq \bar{\psi}_{1}\left(3+2 \bar{\psi}_{3}-2 \underline{\psi}_{1}\right), \\
& 2 \bar{\psi}_{3}+\underline{\psi}_{1} \geq \underline{\psi}_{3}\left(3+2 \underline{\psi}_{1}-2 \bar{\psi}_{3}\right) .
\end{aligned}
$$

Observe that there is some symmetry: (1.11) is invariant under interchanging $\bar{\psi}_{1}, \underline{\psi}_{3}$, and (1.12), (1.13) are interchanged if we interchange $\underline{\psi}_{1}, \bar{\psi}_{3}$ as well as $\bar{\psi}_{1}, \underline{\psi}_{3}$ and reverse inequalities. The same holds for (1.14), (1.15). It will be shown that (1.11) is equivalent to Jarník's relation between $\omega$ and $\hat{\omega}$, and that (1.12), (1.13) are equivalent to Laurent's refinement of Khinchin's transference principle as stated in [6].

However, $\bar{\psi}_{i}$ and $\psi_{i}, i=1,2,3$, do not give sufficient information on the functions $\psi_{1}(Q), \psi_{2}(\bar{Q}), \psi_{3}(Q)$. In fact, we will give a rather precise description of them, and consider this description to be the most interesting part of our investigation. It is this description, which we postpone to Section 6, that will provide the tools for the proof of Theorems 1.5 and 1.6, carried out in Sections 7 and 8.

2. Proof of Theorem 1.1. For any $Q$ there is a space $V(Q)$ of dimension $s$ containing $s$ linearly independent lattice points in $\lambda_{s}(Q) K(Q)$.

Lemma 2.1. Suppose $\lambda_{s}(Q)<\lambda_{s+1}(Q)$ for $Q \geq Q_{0}$. Then $V(Q)$ is unique for $Q \geq Q_{0}$, and in fact is constant: $V(Q)=: S$ for $Q \geq Q_{0}$.

Proof. If $V(Q)$ and $V^{\prime}(Q)$ are two spaces with the above property, their span $V(Q)+V^{\prime}(Q)$ will contain at least $s+1$ independent lattice points in $\lambda_{s}(Q) K(Q)$, so that $\lambda_{s}(Q)=\lambda_{s+1}(Q)$. Hence by our hypothesis, for $Q \geq Q_{0}$ there is a unique space $V(Q)$.

We claim the following continuity property: if some sequence $X_{1}, X_{2}, \ldots$ tends to $X$, where $X_{l} \geq Q_{0}$ and $V\left(X_{l}\right)=S$ for $l=1,2, \ldots$, then $V(X)=S$. For when $Q$ runs through an interval $J$, then $K(Q)$ will be contained in a bounded region of $\mathbb{R}^{n}$, and since $\lambda_{s}(Q)$ is continuous, so will be $\lambda_{s}(Q) K(Q)$. This region will contain only finitely many lattice points. Replacing $P_{1}, P_{2}, \ldots$ by a subsequence if necessary, we may suppose that $V\left(X_{l}\right)$ is 
spanned by fixed independent lattice points $\mathbf{p}_{1}, \ldots, \mathbf{p}_{s}$ lying in $\lambda_{s}\left(X_{l}\right) K\left(X_{l}\right)$. Since $\lambda_{s}(Q)$ is continuous and $K(Q)$ is closed and varies continuously with $Q$, we see that $\mathbf{p}_{j} \in \lambda_{s}(X) K(X)$ for $j=1, \ldots, s$, hence indeed $V(X)=S$.

Given a space $S$ and an interval $J=\left[Q_{0}, Q_{1}\right]$ where $Q_{1}>Q_{0}$, let $J(S)$ consist of the numbers $Q \in J$ with $V(Q)=S$. The sets $J(S)$ cover $J$ and are disjoint by the uniqueness of $V(Q)$. Moreover, they are closed by the continuity property established above. Since $Q \in J$ is bounded, there are only finitely many spaces $S$ having $S=V(Q)$ for some $Q \in J$, hence only finitely many nonempty sets $J\left(S_{1}\right), \ldots, J\left(S_{l}\right)$. But a finite number of nonempty mutually disjoint closed sets can cover $J$ only if there is just one such set. Therefore $J(S)=J$ for some $S$, hence $V(Q)=S$ for $Q \in J$. Since $Q_{1}>Q_{0}$ above was arbitrary, $V(Q)=S$ for $Q \geq Q_{0}$.

Proof of Theorem 1.1. Suppose there was a $Q_{0}$ and a space $S$ as in the preceding lemma. Then $\Lambda_{S}:=\Lambda \cap S$ is a lattice in $S$. Also, $K_{S}(Q):=$ $K(Q) \cap S$ is a symmetric convex body in $S$ whose $s$-dimensional volume we denote by $\operatorname{Vol}_{S}(Q)$. Given $Q \geq Q_{0}$ so that $V(Q)=S$, then (by the uniqueness of $V(Q)) S$ contains independent lattice points $\mathbf{p}_{1}, \ldots, \mathbf{p}_{s}$ with $\mathbf{p}_{j} \in \lambda_{j}(Q) K(Q)$, hence in fact with $\mathbf{p}_{j} \in \Lambda_{S} \cap \lambda_{j}(Q) K_{S}(Q)$ for $1 \leq j \leq s$. By Minkowski's Second Convex Body Theorem applied to $\Lambda_{S}(Q), K_{S}(Q)$, we have

$$
\lambda_{1}(Q) \cdots \lambda_{s}(Q) \geq c_{1}(\Lambda, S, K) / \operatorname{Vol}_{S}(Q) .
$$

On the other hand, applying Minkowski's Theorem to $\Lambda, K(Q)$ yields

$$
\lambda_{1}(Q) \cdots \lambda_{n}(Q) \leq c_{2}(\Lambda) / \operatorname{Vol}(K(Q))=c_{2}(\Lambda) / \operatorname{Vol}(K)=c_{3}(\Lambda, K),
$$

hence $\lambda_{1}(Q) \cdots \lambda_{s}(Q) \leq c_{4}(\Lambda, K)$, so that finally

$$
\operatorname{Vol}_{S}(Q) \geq c_{5}(\Lambda, S, K)>0 \text {. }
$$

If $A$ is as in our hypothesis, we have

$$
\operatorname{Vol}_{S}(Q)=\operatorname{Vol}\left(K_{S}(Q)\right) \leq c_{6}(S) \operatorname{Vol}\left(\pi_{A}(K(Q))\right) .
$$

But if $\mathbf{p} \in K(Q)$, then $\pi_{A}(\mathbf{p})=\left(p_{i_{1}}, \ldots, p_{i_{s}}\right)$ has $\left|p_{i_{j}}\right| \leq c_{7}(K) Q^{\mu_{i_{j}}}(1 \leq$ $j \leq s)$, and therefore

$$
\operatorname{Vol}\left(\pi_{A}(K(Q))\right) \leq c_{8}(K) Q^{\mu_{A}} .
$$

Now (2.2) and $\mu_{A}<0$ yield $\operatorname{Vol}_{S}(Q) \rightarrow 0$ as $Q \rightarrow \infty$, a contradiction to (2.1).

COROLlaRY 2.2. Suppose $1, \xi_{1}, \ldots, \xi_{n-1}$ are linearly independent over $\mathbb{Q}$ and $\mu_{1}+\cdots+\mu_{n}=0$ with $\mu_{i}<0$ for $2 \leq i \leq n$. Let $\lambda_{i}(Q), 1 \leq i \leq n$, be the successive minima with respect to $K(Q)$ and $\Lambda(\xi)$. Then for every $s<n$, there are arbitrarily large values of $Q$ for which $\lambda_{s}(Q)=\lambda_{s+1}(Q)$.

Proof. First suppose that $s=n-1$ and set $A_{0}=\{2, \ldots, n\}$, so that $\mu_{A_{0}}<0$. By Theorem 1.1 it will suffice to show that any $(n-1)$-dimensional 
subspace $S$ spanned by lattice points has $\pi_{A_{0}}(S)=\mathbb{R}^{n-1}=\mathbb{R}^{s}$. Since $\Lambda$ consists of points $\mathbf{p}=\left(x, \xi_{1} x-y_{1}, \ldots, \xi_{n-1} x-y_{n-1}\right)$ with $\left(x, y_{1}, \ldots, y_{n-1}\right)$ $\in \mathbb{Z}^{n}$, the space $S$ will consist of points $\mathbf{p}$ where $\left(x, y_{1}, \ldots, y_{n-1}\right) \in \mathbb{Z}^{n}$ satisfies a nontrivial equation

$$
c x+c_{1} y_{1}+\cdots+c_{n-1} y_{n-1}=0
$$

with integer coefficients. We have to show that $\pi_{A_{0}}(S)$ is not a proper subspace of $\mathbb{R}^{n-1}$, say given by a nontrivial equation

$$
d_{1}\left(\xi_{1} x-y_{1}\right)+\cdots+d_{n-1}\left(\xi_{n-1} x-y_{n-1}\right)=0
$$

with real coefficients. This equation may be rewritten as

$$
\left(d_{1} \xi_{1}+\cdots+d_{n-1} \xi_{n-1}\right) x-d_{1} y_{1}-\cdots-d_{n-1} y_{n-1}=0 .
$$

We have to show that (2.4) is not a consequence of (2.3), i.e. that the respective coefficient vectors are not proportional. If this were the case, we could, after multiplication by a factor, in fact suppose that they were equal, hence $d_{i}=-c_{i}$ for $i=1, \ldots, n-1$ and $c_{1} \xi_{1}+\cdots+c_{n-1} \xi_{n-1}=c$, contradicting the independence of $1, \xi_{1}, \ldots, \xi_{n-1}$.

Now suppose that $\operatorname{dim} S=s$ with $1 \leq s<n-1$. Then $S$ can be embedded in a space $S^{\prime}$ spanned by lattice points with $\operatorname{dim} S^{\prime}=n-1$. We saw that $\pi_{A_{0}}\left(S^{\prime}\right)=\mathbb{R}^{n-1}$, and it follows that $T:=\pi_{A_{0}}(S)$ has dimension $s$. There is some $\left(p_{i_{1}}, \ldots, p_{i_{s}}\right)$-coordinate space of $\mathbb{R}^{n-1}$ such that the projection of $T$ on this space is surjective. Now we have $\pi_{A}(S)=\mathbb{R}^{s}$ with $A=\left\{i_{1}<\cdots<i_{s}\right\}$. Since $\mu_{A}<0$, the corollary follows.

Remarks. (a) Corollary 2.2 applies in particular in the context of Dirichlet's Theorem on simultaneous approximation, where $\mu_{1}=1$ and $\mu_{2}=$ $\cdots=\mu_{n}=-1 /(n-1)$.

(b) The assertion of Corollary 2.2 is not true in general when the numbers $1, \xi_{1}, \ldots, \xi_{n-1}$ are linearly dependent over $\mathbb{Q}$. For instance take $n=3$, $\mu_{1}=1, \mu_{2}=\mu_{3}=-1 / 2$ and $\xi_{1}=\alpha, \xi_{2}=\alpha+1$, where $\alpha$ has bounded partial denominators in its continued fraction expansion. The convergents $p_{\nu} / q_{\nu}, \nu=0,1, \ldots$, to $\alpha$ will have $q_{\nu+1}<c q_{\nu}$ for some $c$. Given large $Q$ there will be some $\nu$ with $c^{-2} Q^{3 / 4} \leq q_{\nu}<q_{\nu+1}<Q^{3 / 4}$. Then

$$
\left|q_{\nu} \alpha-p_{\nu}\right|<q_{\nu}^{-1} \leq c^{2} Q^{-3 / 4}, \quad\left|q_{\nu}(\alpha+1)-p_{\nu}-q_{\nu}\right| \leq c^{2} Q^{-3 / 4},
$$

and the same holds with $\nu+1$ in place of $\nu$. It easily follows that $\lambda_{1}(Q) \leq$ $\lambda_{2}(Q) \leq c^{2} Q^{-1 / 4}$, hence certainly $\lambda_{2}(Q)<\lambda_{3}(Q)$ for large $Q$. Observe that the points $\left(q_{\nu}, q_{\nu} \alpha-p_{\nu}, q_{\nu}(\alpha+1)-p_{\nu}-q_{\nu}\right)$ lie in a space $S$ of dimension 2 .

(c) For the existence of arbitrarily large values of $Q$ for which $\lambda_{s}(Q)=$ $\lambda_{s+1}(Q)$ the fact that two consecutive successive minima are dealt with is crucial: answering a problem raised in [12], Moshchevitin [10] showed that for any $s<n-1$ there exist $\xi_{j} \in[0,1), 1 \leq j \leq n-1$, such that $1, \xi_{1}, \ldots, \xi_{n-1}$ are linearly independent over $\mathbb{Q}, \lambda_{s}(Q) \rightarrow 0$ and $\lambda_{s+2}(Q) \rightarrow \infty$ as $Q \rightarrow \infty$. 
COROLlary 2.3. Suppose $1, \xi_{1}, \ldots, \xi_{n-1}$ are linearly independent over $\mathbb{Q}$ and $\mu_{1}+\cdots+\mu_{n}=0$ with $\mu_{i}>0$ for $2 \leq i \leq n$. Let $\lambda_{i}^{*}(Q), 1 \leq i \leq n$, be the successive minima with respect to $K(Q)$ and $\Lambda^{*}(\xi)$. Then for every $s<n$, there are arbitrarily large values of $Q$ for which $\lambda_{s}^{*}(Q)=\lambda_{s+1}^{*}(Q)$.

Proof. Observe that $x-\xi_{1} y_{1}-\cdots-\xi_{n-1} y_{n-1} \neq 0$ when $\left(x, y_{1}, \ldots, y_{n-1}\right)$ lies in $\mathbb{Q}^{n} \backslash\{\mathbf{0}\}$. So if $A_{1}=\{1\}$, every nonzero space $S$ spanned by lattice points has $\pi_{A_{1}}(S) \neq \mathbf{0}$, in fact $\pi_{A_{1}}(S)=\mathbb{R}$. Hence when $\operatorname{dim} S=s<n$, there is some $A=\left\{1<i_{2}<\cdots<i_{s}\right\}$ with $\pi_{A}(S)=\mathbb{R}^{s}$. Since $\mu_{A}=$ $-\sum_{i \notin A} \mu_{i}<0$, the conclusion follows via Theorem 1.1.

REMARK. Corollary 2.3 applies in particular in the context of Dirichlet's Theorem on linear forms, where $\mu_{1}=-1$ and $\mu_{2}=\cdots=\mu_{n}=1 /(n-1)$.

3. $\bar{\psi}_{i}, \underline{\psi}_{i}$ and the proof of Theorem 1.2. For $A \subseteq\{1, \ldots, n\}$ set

$$
\psi_{A}(Q):=\sum_{i \in A} \psi_{i}(Q), \quad \bar{\psi}_{A}:=\limsup _{Q \rightarrow \infty} \psi_{A}(Q), \quad \underline{\psi}_{A}:=\liminf _{Q \rightarrow \infty} \psi_{A}(Q) .
$$

Lemma 3.1. When $A, B$ are complementary nonempty subsets of $\{1, \ldots, n\}$, we have

and moreover

$$
\bar{\psi}_{A}+\underline{\psi}_{B}=0
$$

$$
\sum_{i \in A} \underline{\psi}_{i} \leq \underline{\psi}_{A} \leq|A| \max _{i \in A} \underline{\psi}_{i}, \quad|A| \min _{i \in A} \bar{\psi}_{i} \leq \bar{\psi}_{A} \leq \sum_{i \in A} \bar{\psi}_{i} .
$$

Proof. By (1.4) we have $\psi_{A}(Q)+\psi_{B}(Q) \rightarrow 0$ as $Q \rightarrow \infty$. So when $\epsilon>0$ and $Q$ is large, we have $\psi_{A}(Q)<\bar{\psi}_{A}+\epsilon$ and $\psi_{B}(Q)>-\bar{\psi}_{A}-2 \epsilon$. Since $\epsilon>0$ is arbitrary, this yields $\underline{\psi}_{B} \geq-\bar{\psi}_{A}$, i.e. $\bar{\psi}_{A}+\underline{\psi}_{B} \geq 0$. When $\epsilon>0$ and $Q$ is large, we have $\psi_{B}(Q)>\underline{\psi}_{B}-\epsilon$ and $\psi_{A}(Q)<-\underline{\psi}_{B}+2 \epsilon$, hence eventually $\bar{\psi}_{A}+\underline{\psi}_{B} \leq 0$. This proves the first assertion.

The estimates

$$
\sum_{i \in A} \underline{\psi}_{i} \leq \underline{\psi}_{A}, \quad \bar{\psi}_{A} \leq \sum_{i \in A} \bar{\psi}_{i}
$$

follow immediately from the definitions.

If $A=\left\{i_{1}<\cdots<i_{s}\right\}$, there are for $\epsilon>0$ arbitrarily large values of $Q$ with $\psi_{i_{s}}(Q) \leq \underline{\psi}_{i_{s}}+\epsilon$, hence

$$
\sum_{i \in A} \psi_{i}(Q) \leq|A|\left(\underline{\psi}_{i_{s}}+\epsilon\right) \leq|A| \max _{i \in A} \underline{\psi}_{i}+|A| \epsilon .
$$

On the other hand, for any $\epsilon>0$ there are arbitrarily large values of $Q$ with $\psi_{i_{s}}(Q) \geq \bar{\psi}_{i_{s}}-\epsilon$, hence

$$
\sum_{i \in A} \psi_{i}(Q) \geq|A|\left(\bar{\psi}_{i_{s}}-\epsilon\right) \geq|A| \min _{i \in A} \bar{\psi}_{i}-|A| \epsilon
$$

and the lemma follows. 
Proof of Theorem 1.2. Taking $A=\{i\}, B=\{1, \ldots, n\} \backslash\{i\}$ we have

$$
\begin{aligned}
& 0=\underline{\psi}_{A}+\bar{\psi}_{B} \leq \underline{\psi}_{i}+\left(\bar{\psi}_{1}+\cdots+\bar{\psi}_{i-1}+\bar{\psi}_{i+1}+\cdots+\bar{\psi}_{n}\right), \\
& 0=\bar{\psi}_{A}+\underline{\psi}_{B} \geq \bar{\psi}_{i}+\left(\underline{\psi}_{1}+\cdots+\underline{\psi}_{i-1}+\underline{\psi}_{i+1}+\cdots+\underline{\psi}_{n}\right) .
\end{aligned}
$$

4. The functions $L_{1}, \ldots, L_{n}$ and proof of Theorems 1.3 and 1.4. We now specialize to the situation of Dirichlet's Theorem on simultaneous approximation. Thus $\Lambda(\xi)$ consists of points $\mathbf{p}(\mathbf{x})=\left(x, \xi_{1} x-y_{1}, \ldots\right.$, $\left.\xi_{n-1} x-y_{n-1}\right)$ where $1, \xi_{1}, \ldots, \xi_{n-1}$ are linearly independent over $\mathbb{Q}$ and $\mathbf{x}=\left(x, y_{1}, \ldots, y_{n-1}\right)$ runs through $\mathbb{Z}^{n}$. Further, $\mathcal{C}(Q)$ consists of points $\mathbf{p}=\left(p_{1}, \ldots, p_{n}\right)$ with $\left|p_{1}\right| \leq Q$ and $\left|p_{i}\right| \leq Q^{-1 /(n-1)}(i=2, \ldots, n)$. Therefore $\mathbf{p}(\mathbf{x})$ lies in $\lambda \mathcal{C}(Q)$ precisely if

$$
|x| \leq \lambda Q, \quad\left|\xi_{j} x-y_{j}\right| \leq \lambda Q^{-1 /(n-1)} \quad \text { for } 2 \leq j \leq n .
$$

Hence $\lambda_{i}(Q)(i=1, \ldots, n)$ is the least value of $\lambda$ such that there are $i$ independent points $\mathbf{x}$ for which the above system of inequalities is satisfied. If $\lambda_{1}(Q) \leq c / Q$, then the above $\mathbf{x}$ has $|x| \leq c$, and $\left|\xi_{1} x-y_{1}\right| \leq$ $\lambda_{1}(Q) Q^{-1 /(n-1)} \leq c Q^{-n /(n-1)}$ has no solution $y_{1}$ for large $Q$. Therefore

$$
\lambda_{1}(Q) \geq f_{1}(Q) / Q
$$

where $f_{1}(Q)$ tends to $\infty$ as $Q \rightarrow \infty$.

Given $\mathbf{x} \in \mathbb{Z}^{n} \backslash\{\mathbf{0}\}$ we let $\lambda_{\mathbf{x}}(Q)$ be the least $\lambda>0$ with $\mathbf{p}(\mathbf{x}) \in \lambda \mathcal{C}(Q)$. Then for any $Q>1$ and $i \in\{1, \ldots, n\}$ we have $\lambda_{i}(Q)=\lambda_{\mathbf{x}}(Q)$ for some $\mathbf{x}$ depending on $Q$ and $i$. In particular,

$$
\lambda_{1}(Q)=\min _{\mathbf{x} \in \mathbb{Z}^{n} \backslash\{\mathbf{0}\}} \lambda_{\mathbf{x}}(Q) .
$$

Clearly,

$$
\lambda_{\mathbf{x}}(Q)=\max \left\{|x| Q^{-1}, \max _{1 \leq j \leq n-1}\left\{\left|\xi_{j} x-y_{j}\right|\right\} Q^{1 /(n-1)}\right\} .
$$

Since $\lambda_{\mathbf{x}}(Q) \geq Q^{1 /(n-1)}$ (otherwise, we will restrict to points $\mathbf{x}$ with $x \neq 0$ ), and since $\lambda_{\mathbf{x}}(Q)=\lambda_{-\mathbf{x}}(Q)$, we may suppose $x>0$.

It will be convenient to replace $Q$ as well as $\lambda_{i}(Q), \lambda_{\mathbf{x}}(Q)$ by their logarithms. Accordingly we set

$$
\begin{aligned}
q & :=\log Q, \\
\chi_{i}(q) & :=\psi_{i}\left(e^{q}\right), \\
L_{i}(q) & :=\log \lambda_{i}\left(e^{q}\right)=q \chi_{i}(q), \\
L_{\mathbf{x}}(q) & :=\log \lambda_{\mathbf{x}}\left(e^{q}\right),
\end{aligned}
$$

and with these notations we obtain

$$
L_{\mathbf{x}}(q)=\max \left\{\log |x|-q, \max _{1 \leq j \leq n-1}\left\{\log \left|\xi_{j} x-y_{j}\right|\right\}+\frac{q}{n-1}\right\} .
$$


Thus $L_{\mathbf{x}}(q)$ has its minimum at some $q=q(\mathbf{x})$, and is linear with slope -1 for $q \leq q(\mathbf{x})$ and slope $1 /(n-1)$ for $q \geq q(\mathbf{x})$. When $Q$ lies in a bounded range, there will be only finitely many $\mathbf{x}$ having $\lambda_{i}(Q)=\lambda_{\mathbf{x}}(Q)$ for some $i$. Therefore the functions $L_{i}$ will be continuous, piecewise linear, with slopes among -1 and $1 /(n-1)$.

When $\epsilon>0$ and $q$ is large, then $\underline{\psi}_{i}-\epsilon<\chi_{i}(q)<\bar{\psi}_{i}+\epsilon$, and therefore

$$
q \underline{\psi}_{i}-o(q) \leq L_{i}(q) \leq q \bar{\psi}_{i}+o(q) .
$$

Set $\mathbf{x}_{1}=\left(1, y_{2}, \ldots, y_{n}\right)$ with $\left|\xi_{j}-y_{j}\right|<1 / 2(1 \leq j<n)$. It is easily seen that in some interval $1<Q<Q_{0}$, the function $\lambda_{1}(Q)$ equals $\lambda_{\mathbf{x}_{1}}(Q)$, and is decreasing. Since $L_{1}$ is piecewise linear with only finitely many pieces within any bounded interval, there will be numbers $0<q_{1}<q_{2}<\cdots$ tending to infinity such that $L_{1}$ has its local minima precisely at $q_{k}, k=1,2, \ldots$ There will be points $\mathbf{x}_{k}$ with $L_{1}\left(q_{k}\right)=L_{\mathbf{x}_{k}}\left(q_{k}\right)$, and in fact there will be a neighborhood of $q_{k}$ with $L_{1}(q)=L_{\mathbf{x}_{k}}(q)$. More precisely, if $q_{0,1}=0$ and $q_{k, k+1}$ for $k>0$ is the $q$-coordinate of the point of intersection of the graphs of $L_{\mathbf{x}_{k}}$ and $L_{\mathbf{x}_{k+1}}$, then for $k>0$,

$$
L_{1}(q)=L_{\mathbf{x}_{k}}(q) \quad \text { for } q_{k-1, k} \leq q \leq q_{k, k+1},
$$

which also holds for $k=1$ if we define $L_{1}(0)=0$.

$L_{1}$ will decrease with slope -1 in $\left[q_{k-1, k}, q_{k}\right]$ and increase with slope $1 /(n-1)$ in $\left[q_{k}, q_{k, k+1}\right]$. Thus $L_{1}$ will have its local maxima at $q_{k, k+1}, k=$ $1,2, \ldots$ Moreover,

$$
L_{1}\left(q_{k, k+1}\right)=L_{\mathbf{x}_{k}}\left(q_{k, k+1}\right)=L_{\mathbf{x}_{k+1}}\left(q_{k, k+1}\right),
$$

and since $\mathbf{x}_{k}, \mathbf{x}_{k+1}$ are easily seen to be linearly independent we have

$$
L_{2}\left(q_{k, k+1}\right)=L_{1}\left(q_{k, k+1}\right) .
$$

So if $L_{1}$ has a local maximum at $q$ (hence $q=q_{k, k+1}$ for some $k$ ), then $L_{1}(q)=L_{2}(q)$.

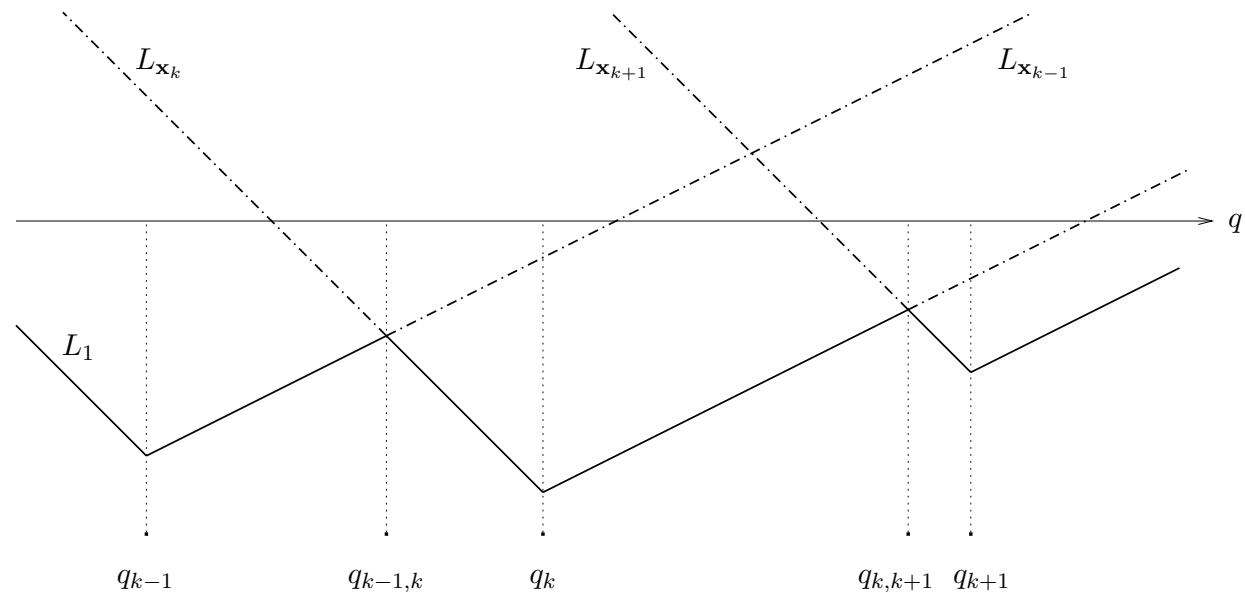


REMARK. It is well known, and follows from our arguments in Section 2, that for $n \geq 3$ there are arbitrarily large $k$ with $\mathbf{x}_{k}, \mathbf{x}_{k+1}, \mathbf{x}_{k+2}$ linearly independent. On the other hand, according to Moshchevitin [9], when $n>3$, then there are numbers $1, \xi_{1}, \ldots, \xi_{n-1}$ linearly independent over $\mathbb{Q}$ such that $\mathbf{x}_{k}, \mathbf{x}_{k+1}, \ldots, \mathbf{x}_{k+n-1}$ for every large $k$ span a space of dimension at most 3 .

The function $L_{1}^{*}$ will have its local minima at numbers $q_{1}^{*}<q_{2}^{*}<\cdots$, and $L_{1}^{*}\left(q_{l}^{*}\right)=L_{\mathbf{x}_{l}^{*}}^{*}\left(q_{l}^{*}\right)$ for certain points $\mathbf{x}_{1}^{*}, \mathbf{x}_{2}^{*}, \ldots$ For suitably defined $q_{l, l+1}^{*}$ with $q_{l}^{*}<q_{l, l+1}^{*}<q_{l+1}^{*}$ we will have

$$
L_{1}^{*}(q)=L_{\mathbf{x}_{l}^{*}}^{*}(q) \quad \text { for } q_{l-1, l}^{*} \leq q \leq q_{l, l+1}^{*}
$$

with $L_{1}^{*}$ having slope 1 in $\left[q_{l-1, l}^{*}, q_{l}^{*}\right]$ and slope $-1 /(n-1)$ in $\left[q_{l}^{*}, q_{l, l+1}^{*}\right]$. Moreover, $L_{2}^{*}\left(q_{l, l+1}^{*}\right)=L_{1}^{*}\left(q_{l, l+1}^{*}\right)$. So when $L_{1}^{*}$ has a local maximum at $q$, then $L_{1}^{*}(q)=L_{2}^{*}(q)$.

Mahler's inequality $\lambda_{i}^{*}(Q) \lambda_{n+1-i}(Q) \asymp 1$, where the implied constants depend on $n$ only, gives

$$
\left|L_{i}^{*}(q)+L_{n+1-i}(q)\right|<c \quad(i=1, \ldots, n)
$$

where the constant $c$ depends on $n$ only. Further, $L_{i}(q)=q \psi_{i}\left(e^{q}\right)$, so $(1.4)$ yields

$$
\left|L_{1}(q)+\cdots+L_{n}(q)\right|<c
$$

if $c=c(n)$ was chosen large enough, and we also have

$$
\left|L_{1}^{*}(q)+\cdots+L_{n}^{*}(q)\right|<c .
$$

It is not hard to see that $\lambda_{1}^{*}(Q)>f_{1}^{*}(Q) / Q^{1 /(n-1)}$ with $f_{1}^{*}(Q)$ tending to infinity with $Q$, in analogy to (4.1), and therefore by Mahler's inequality $\lambda_{n}(Q) \asymp \lambda_{1}^{*}(Q)^{-1}<Q^{1 /(n-1)} / f_{1}^{*}(Q)$. But this, together with (4.1), tells us that

$$
L_{1}(q)>-q+g_{1}(Q) \quad \text { and } \quad L_{n}(q)<\frac{q}{n-1}-g_{n}(Q)
$$

with $g_{1}(Q), g_{n}(Q)$ both tending to infinity.

We had

$$
\psi_{i}(Q)=\log \lambda_{i}(Q) / \log Q \quad \text { and } \quad \chi_{i}(q)=\psi_{i}\left(e^{q}\right)=L_{i}(q) / q
$$

so that

$$
\begin{aligned}
& \bar{\psi}_{i}=\limsup \psi_{i}(Q)=\limsup \chi_{i}(q), \\
& \underline{\psi}_{i}=\liminf \psi_{i}(Q)=\liminf \chi_{i}(q) .
\end{aligned}
$$

We now set

$$
\psi_{i}^{*}(Q)=\log \lambda_{i}^{*}(Q) / \log Q \quad \text { and } \quad \chi_{i}^{*}(q)=\psi_{i}^{*}\left(e^{q}\right)=L_{i}^{*}(q) / q,
$$


so that

$$
\begin{aligned}
& \bar{\psi}_{i}^{*}=\lim \sup \psi_{i}^{*}(Q)=\lim \sup \chi_{i}^{*}(q), \\
& \underline{\psi}_{i}^{*}=\liminf \psi_{i}^{*}(Q)=\liminf \chi_{i}^{*}(q) .
\end{aligned}
$$

Inequality (4.6) yields $\left|\psi_{i}^{*}(q)+\psi_{n+1-i}(q)\right|<c / \log Q$, so that

$$
\bar{\psi}_{i}^{*}+\underline{\psi}_{n+1-i}=0 \quad \text { and } \quad \underline{\psi}_{i}^{*}+\bar{\psi}_{n+1-i}=0 \quad(i=1, \ldots, n) .
$$

LEMMA 4.1. If $i \neq j$ and $L_{i}$ has slope -1 in some interval, then for $q, q^{\prime}$ in that interval,

$$
L_{j}(q)-L_{j}\left(q^{\prime}\right)=\frac{q-q^{\prime}}{n-1}+O(1) .
$$

Here and throughout, the implied constants in $\asymp, O(\ldots), \ll$ depend only on $n$.

Proof. Say $q>q^{\prime}$. Each $L_{l}$ has slopes $-1,1 /(n-1)$ so that

$$
L_{l}(q)-L_{l}\left(q^{\prime}\right) \leq \frac{q-q^{\prime}}{n-1} \quad(1 \leq l \leq n) .
$$

By (4.7) and (4.12),

$$
\begin{aligned}
L_{j}(q)-L_{j}\left(q^{\prime}\right) & \geq-\sum_{l \neq j}\left(L_{l}(q)-L_{l}\left(q^{\prime}\right)\right)+O(1) \\
& =-\left(L_{i}(q)-L_{i}\left(q^{\prime}\right)\right)-\sum_{l \neq i, j}\left(L_{l}(q)-L_{l}\left(q^{\prime}\right)\right)+O(1) \\
& \geq q-q^{\prime}-(n-2) \frac{q-q^{\prime}}{n-1}+O(1)=\frac{q-q^{\prime}}{n-1}+O(1) .
\end{aligned}
$$

The lemma follows from this and from (4.12) with $l=j$.

Proof of Theorem 1.3. For $k \geq 2$ we have

$$
L_{1}\left(q_{k-1, k}\right)=L_{2}\left(q_{k-1, k}\right) \leq 0, \quad L_{1}\left(q_{k}\right)=L_{1}\left(q_{k-1, k}\right)-\left(q_{k}-q_{k-1}\right)
$$

and by Lemma 4.1,

Therefore

$$
L_{2}\left(q_{k}\right)=L_{2}\left(q_{k-1, k}\right)+\frac{q_{k}-q_{k-1, k}}{n-1}+O(1) .
$$

$$
n L_{2}\left(q_{k-1, k}\right)=(n-1) L_{2}\left(q_{k}\right)+L_{1}\left(q_{k}\right)+O(1) .
$$

Since both sides are $\leq 0$, and $q_{k-1, k} \leq q_{k}$,

$$
n \psi_{2}\left(q_{k-1, k}\right) \leq(n-1) \psi_{2}\left(q_{k}\right)+\psi_{1}\left(q_{k}\right)+O\left(1 / q_{k-1, k}\right),
$$

hence we may infer that

$$
\begin{aligned}
n \underline{\psi}_{2} & \leq n \liminf \psi_{2}\left(q_{k-1, k}\right) \leq(n-1) \lim \sup \psi_{2}\left(q_{k}\right)+\liminf \psi_{1}\left(q_{k}\right) \\
& =(n-1) \bar{\psi}_{2}+\underline{\psi}_{1},
\end{aligned}
$$

which is (1.7). 
The proof of (1.8) is dual to that of (1.7): by (4.11) we need to show that

$$
n \underline{\psi}_{2}^{*} \leq(n-1) \bar{\psi}_{2}^{*}+\bar{\psi}_{1}^{*} \text {. }
$$

Lemma 4.1 is replaced by the fact that if $i \neq j$ and $L_{i}^{*}$ has slope 1 , then

$$
L_{j}^{*}(q)-L_{j}^{*}\left(q^{\prime}\right)=\frac{q^{\prime}-q}{n-1}+O(1) .
$$

Instead of (4.13) we have

$$
n L_{2}^{*}\left(q_{k, k+1}\right)=(n-1) L_{2}^{*}\left(q_{k}\right)+L_{1}^{*}\left(q_{k}\right)+O(1) ;
$$

now we proceed as in the first part of the proof.

Proof of Theorem 1.4. If $\theta>\underline{\psi}_{1}$, then

$$
\begin{aligned}
|x| & \leq Q^{1+\theta}, \\
\left|\xi_{i} x-y_{i}\right| & \leq Q^{-1 /(n-1)+\theta} \quad \text { for } i=1, \ldots, n-1
\end{aligned}
$$

has a nonzero integer solution for certain arbitrarily large values of $Q$. Setting

$$
X=Q^{1+\theta} \quad \text { and } \quad \eta=-1+\frac{n}{(n-1)(1+\theta)}
$$

the system (4.14) becomes

$$
|x| \leq X, \quad\left|\xi_{i} x-y_{i}\right| \leq X^{-\eta} \quad \text { for } i=1, \ldots, n-1 .
$$

Therefore $\omega \geq \eta$, and since $\theta$ is arbitrarily close to $\underline{\psi}_{1}$, we obtain

$$
\omega \geq-1+\frac{n}{(n-1)\left(1+\underline{\psi}_{1}\right)},
$$

hence

$$
(1+\omega)\left(1+\underline{\psi}_{1}\right) \geq \frac{n}{n-1} .
$$

When $\eta<\omega,(4.15)$ has a solution for certain arbitrarily large numbers $X$. Setting

$$
Q=X^{1 /(1+\eta)} \quad \text { and } \quad \theta=-1+\frac{n}{(n-1)(1+\eta)},
$$

the system (4.15) becomes (4.14) and hence $\underline{\psi}_{1} \leq \theta$. As $\eta$ can be taken arbitrarily close to $\omega$, we obtain

$$
\underline{\psi}_{1} \leq-1+\frac{n}{(n-1)(1+\omega)} \Leftrightarrow(1+\omega)\left(1+\underline{\psi}_{1}\right) \leq \frac{n}{n-1} .
$$

Therefore the first expression in (1.9) equals $n /(n-1)$, and the proof for the second one is similar.

We had $\psi_{i}^{*}(Q)=\log \lambda_{i}^{*}(Q) / \log Q$, hence $\lambda_{i}^{*}(Q)=Q^{\psi_{i}^{*}(Q)}$, and we now define $\pi_{i}(Q)$ by $\nu_{i}(Q)=Q^{\pi_{i}(Q)}$. By $(1.1),\left|\pi_{i}(Q)-\psi_{i}^{*}(Q)\right|<n / \log Q$, so that

$$
\bar{\pi}_{i}:=\lim \sup \pi_{i}(Q)=\bar{\psi}_{i}^{*} \quad \text { and } \quad \underline{\pi}_{i}:=\liminf \pi_{i}(Q)=\underline{\psi}_{i}^{*} .
$$


Therefore, by $(4.11), \bar{\psi}_{n}=-\underline{\psi}_{1}^{*}=-\underline{\pi}_{1}$ and $\underline{\psi}_{n}=-\bar{\psi}_{1}^{*}=-\bar{\pi}_{1}$, so that in order to establish (1.10) we need to prove

$$
\left(\omega^{*}+1\right)\left(\frac{1}{n-1}+\underline{\pi}_{1}\right)=\left(\hat{\omega}^{*}+1\right)\left(\frac{1}{n-1}+\bar{\pi}_{1}\right)=\frac{n}{n-1} .
$$

This is proved similarly to (1.9).

Laurent in [6] gives best possible upper and lower bounds for $\omega, \hat{\omega}, \omega^{*}, \hat{\omega}^{*}$. Since

$$
\frac{1}{n-1} \leq \omega \leq \infty \quad \text { and } \quad \frac{1}{n-1} \leq \hat{\omega} \leq 1
$$

are best possible, (1.9) gives the best possible bounds

$$
-1 \leq \underline{\psi}_{1} \leq 0 \quad \text { and } \quad-\frac{n-2}{2(n-1)} \leq \bar{\psi}_{1} \leq 0 .
$$

Further, $n-1 \leq \omega^{*} \leq \infty$ and $n-1 \leq \hat{\omega}^{*} \leq \infty$, so that by (1.10),

$$
0 \leq \bar{\psi}_{n} \leq \frac{1}{n-1} \quad \text { and } \quad 0 \leq \underline{\psi}_{n} \leq \frac{1}{n-1}
$$

Again these estimates are best possible.

REMARKS. (a) Jarník's identity $\hat{\omega}=\hat{\omega}^{*}-1 / \hat{\omega}^{*}$ is equivalent to equation (1.11) of Theorem 1.5, for this identity is in turn equivalent to

$$
\begin{aligned}
\hat{\omega}=\frac{\hat{\omega}^{*}-1}{\hat{\omega}^{*}} & \Leftrightarrow \hat{\omega}+1=2-\frac{1}{\hat{\omega}^{*}} \Leftrightarrow \frac{3}{2 \bar{\psi}_{1}+2}=2+\frac{2 \underline{\psi}_{3}-1}{2 \underline{\psi}_{3}+2} \\
& \Leftrightarrow \frac{1}{\bar{\psi}_{1}+1}=\frac{2 \underline{\psi}_{3}+1}{\underline{\psi}_{3}+1} \Leftrightarrow \bar{\psi}_{1}+\underline{\psi}_{3}+2 \bar{\psi}_{1} \underline{\psi}_{3}=0 .
\end{aligned}
$$

(b) Khinchin's transference principle ([5]) says that

$$
\omega \geq \frac{\omega^{*}}{(n-2) \omega^{*}+n-1} \quad \text { and } \quad \omega^{*} \geq(n-1) \omega+n-2 .
$$

The first of these inequalities yields

$$
\omega+1 \geq \frac{\omega^{*}+(n-2) \omega^{*}+n-1}{(n-2) \omega^{*}+n-1}=\frac{n-1}{n-2+1 /\left(\omega^{*}+1\right)},
$$

hence by Theorem 1.4,

$$
\frac{n /(n-1)}{1+\underline{\psi}_{1}} \geq \frac{n-1}{n-2+\left(1 /(n-1)-\bar{\psi}_{n}\right)(n-1) / n}=\frac{n-1}{n-1-\bar{\psi}_{n}},
$$

so that $n-1-\bar{\psi}_{n} \geq(n-1)\left(1+\underline{\psi}_{1}\right)$, hence $(n-1) \underline{\psi}_{1}+\bar{\psi}_{n} \leq 0$.

The second relation in Khinchin's transference principle leads to $\underline{\psi}_{1}+$ $(n-1) \bar{\psi}_{n} \geq 0$. Conversely, these relations imply Khinchin's transference principle. Observe that they are consequences of Theorem 1.2. 
(c) Laurent's refinement of Khinchin's relations between the approximation exponents in [6] states that

$$
\frac{\omega^{*}\left(\hat{\omega}^{*}-1\right)}{\omega^{*}+\hat{\omega}^{*}} \leq \omega \leq \frac{\omega^{*}-\hat{\omega}^{*}+1}{\hat{\omega}^{*}},
$$

which is equivalent to the pair of inequalities (1.12), (1.13) of Theorem 1.6. For expressing the classical approximation constants in terms of $\underline{\psi}_{1}, \underline{\psi}_{3}$, $\bar{\psi}_{3}$ using the equations from Theorem 1.4, a lengthy but straightforward computation translates Laurent's inequalities into

$$
\frac{3 \underline{\psi}_{3}-2 \bar{\psi}_{3}-2 \underline{\psi}_{3} \bar{\psi}_{3}}{1-2 \underline{\psi}_{3}} \leq \underline{\psi}_{1} \leq \frac{-3 \underline{\psi}_{3}-\bar{\psi}_{3}-4 \underline{\psi}_{3} \bar{\psi}_{3}}{2+2 \underline{\psi}_{3}},
$$

which turns out to be precisely equivalent to (1.14), (1.15) and hence to (1.12), (1.13).

5. The functions $\tilde{L}_{1}, \tilde{L}_{2}, \tilde{L}_{3}$. We now turn to the case $n=3$ in the context of Dirichlet's Theorem on simultaneous approximation. It would be nice if we had $L_{1}(q)+L_{2}(q)+L_{3}(q)=0$ in place of the case $n=3$ of (4.7). We therefore introduce the new triple of functions $\left(\tilde{L}_{1}, \tilde{L}_{2}, \tilde{L}_{3}\right)$ with

$$
\tilde{L}_{1}:=L_{1}, \quad \tilde{L}_{2}:=L_{1}^{*}-L_{1}, \quad \tilde{L}_{3}:=-L_{1}^{*},
$$

whose properties will now be investigated.

LEMma 5.1. If $c$ is the constant of (4.6)-(4.8), then:

(i) $\tilde{L}_{1}+\tilde{L}_{2}+\tilde{L}_{3}=0$.

(ii) $\left|\tilde{L}_{i}(q)-L_{i}(q)\right|<2 c$ for $i=1,2,3$.

(iii) $\tilde{L}_{2}(q)>\tilde{L}_{1}(q)-4 c$ and $\tilde{L}_{3}(q)>\tilde{L}_{2}(q)-4 c$.

(iv) $\tilde{L}_{1}, \tilde{L}_{3}$ have slopes alternating between $-1,1 / 2$, whereas $\tilde{L}_{2}$ has slopes among $-1,1 / 2,2$.

(v) An interval where $\tilde{L}_{2}$ has slope 2 has length less than $3 c$.

(vi) In an interval where $\tilde{L}_{3}(q)-\tilde{L}_{2}(q)>4 c$ the function $\tilde{L}_{3}$ has no local minimum, and in an interval where $\tilde{L}_{2}(q)-\tilde{L}_{1}(q)>4 c$ the function $\tilde{L}_{1}$ has no local maximum.

(vii) If $\{i, j, k\}=\{1,2,3\}$ and $\tilde{L}_{i}$ has slope $1 / 2$ in some interval, then $\frac{1}{2}\left(\tilde{L}_{j}+\tilde{L}_{k}\right)$ will have slope $-1 / 4$ in this interval. $\tilde{L}_{j}, \tilde{L}_{k}$ will alternate having respective slopes $1 / 2,-1$ and $-1,1 / 2$, hence their graphs will zigzag around a line with slope $-1 / 4$.

(viii) Suppose $i \neq j$ in $\{1,2,3\}$. If $\tilde{L}_{i}$ has slope -1 in some interval, then

$$
\left|\tilde{L}_{j}(q)-\tilde{L}_{j}\left(q^{\prime}\right)-\frac{1}{2}\left(q-q^{\prime}\right)\right|<4 c
$$

for $q, q^{\prime}$ in that interval. 
Proof. (i) is obvious from the definition of $\tilde{L}_{i}(i=1,2,3)$.

For (ii) observe that $\tilde{L}_{1}-L_{1}=0, \tilde{L}_{3}-L_{3}=-\left(L_{1}^{*}+L_{3}\right)$ and $\tilde{L}_{2}-L_{2}=$ $L_{1}^{*}-L_{1}-L_{2}=\left(L_{1}^{*}+L_{3}\right)-\left(L_{1}+L_{2}+L_{3}\right)$. Now apply (4.7) and (4.6).

Next, (iii) follows from (ii) and the fact that

$$
\begin{aligned}
& \tilde{L}_{2}-\tilde{L}_{1}=\tilde{L}_{2}-L_{1}=\left(L_{2}-L_{1}\right)+\left(\tilde{L}_{2}-L_{2}\right) \geq \tilde{L}_{2}-L_{2}, \\
& \tilde{L}_{3}-\tilde{L}_{2}=\left(L_{3}-L_{2}\right)+\left(\tilde{L}_{3}-L_{3}\right)+\left(L_{2}-\tilde{L}_{2}\right) \geq\left(\tilde{L}_{3}-L_{3}\right)+\left(L_{2}-\tilde{L}_{2}\right) .
\end{aligned}
$$

Concerning (iv) note that $\tilde{L}_{1}=L_{1}$ has slopes $-1,1 / 2, L_{1}^{*}$ has slopes $1,-1 / 2$, so that $\tilde{L}_{3}$ has slopes $-1,1 / 2$. The assertion now follows from (i) above.

(v) When $\tilde{L}_{2}$ has slope 2 in $\left[q^{\prime}, q\right]$, then

$$
q-q^{\prime}=\frac{1}{2}\left(\tilde{L}_{2}(q)-\tilde{L}_{2}\left(q^{\prime}\right)\right)<\frac{1}{2}\left(L_{2}(q)-L_{2}\left(q^{\prime}\right)\right)+2 c \leq \frac{1}{4}\left(q-q^{\prime}\right)+2 c,
$$

hence $q-q^{\prime}<\frac{8}{3} c<3 c$.

(vi) Suppose $\tilde{L}_{3}-\tilde{L}_{2}>4 c$ in some interval. Observe that

$$
\tilde{L}_{3}-\tilde{L}_{2}-\left(L_{2}^{*}-L_{1}^{*}\right)=-\tilde{L}_{2}-L_{2}^{*}=-\left(L_{2}+L_{2}^{*}\right)+\left(L_{2}-\tilde{L}_{2}\right) .
$$

Combining this with (4.6) and (ii) implies $L_{2}^{*}-L_{1}^{*} \neq 0$, i.e. $L_{2}^{*} \neq L_{1}^{*}$. Therefore $L_{1}^{*}$ has no local maximum, and $\tilde{L}_{3}=-L_{1}^{*}$ no local minimum in the interval. When $\tilde{L}_{2}-\tilde{L}_{1}>4 c$, then $L_{2}(q)-L_{1}(q)>\tilde{L}_{2}-\tilde{L}_{1}-2 c>0$, hence $L_{2}(q) \neq L_{1}(q)$, so $\tilde{L}_{1}=L_{1}$ has no local maximum.

(vii) is fairly obvious.

(viii) is essentially the case $n=3$ of Lemma 4.1 .

6. Top and bottom intervals. It may happen that, for all large $q$,

$$
\tilde{L}_{3}(q)-\tilde{L}_{1}(q) \leq C,
$$

where $C$ is a constant to be specified below. Then $\underline{\psi}_{i}=\bar{\psi}_{i}=0$ for $i=1,2,3$ and (1.11)-(1.13) trivially hold.

The other extreme is when

$$
\tilde{L}_{3}(q)-\tilde{L}_{1}(q)>C
$$

for all large $q$. We will treat this case in detail. It is also possible that all large $q$ lie in a sequence of intervals which alternate between $q$ 's in the two extremal cases. In this case $\bar{\psi}_{1}=\underline{\psi}_{3}=0$, and this also implies $(1.11),(1.12)$ and (1.13). In large intervals where $\tilde{L}_{3}(q)-\tilde{L}_{1}(q)>C$, the situation is essentially the same as if the equality holds for all large $q$, so we will not elaborate on this case.

We will now suppose (6.1) holds; we set $\gamma=4 c$, where $c$ is the absolute constant of the last section. There are arbitrarily large values of $p$ with $L_{3}(p)=L_{2}(p)$ and hence $\tilde{L}_{3}(p)-\tilde{L}_{2}(p)<\gamma$, so $(6.1)$ implies $\tilde{L}_{2}(p)-\tilde{L}_{1}(p)>$ $C-\gamma>\gamma$, provided $C>2 \gamma$. Also, there are arbitrarily large numbers $p^{*}$ 
with $\tilde{L}_{2}\left(p^{*}\right)-\tilde{L}_{1}\left(p^{*}\right)<\gamma$, hence $\tilde{L}_{3}\left(p^{*}\right)-\tilde{L}_{2}\left(p^{*}\right)>\gamma$. So by the Intermediate Value Theorem there are arbitrarily large values of $p$ with

$$
\tilde{L}_{3}(p)-\tilde{L}_{2}(p)=\gamma,
$$

as well as arbitrarily large numbers $p^{*}$ with

$$
\tilde{L}_{2}\left(p^{*}\right)-\tilde{L}_{1}\left(p^{*}\right)=\gamma \text {. }
$$

If $p, p^{*}$ are such numbers, then $\tilde{L}_{3}\left(p^{*}\right)-\tilde{L}_{2}\left(p^{*}\right)>C-\gamma$ and

$$
\tilde{L}_{3}\left(p^{*}\right)-\tilde{L}_{2}\left(p^{*}\right)-\left(\tilde{L}_{3}(p)-\tilde{L}_{2}(p)\right) \leq 3\left|p-p^{*}\right|,
$$

so that $\tilde{L}_{3}(p)-\tilde{L}_{2}(p)>C-\gamma-3\left|p-p^{*}\right|$, which together with $\tilde{L}_{3}(p)-\tilde{L}_{2}(p)$ $=\gamma$ yields $3\left|p-p^{*}\right|>C-2 \gamma$, hence

$$
\left|p-p^{*}\right|>C / 3-2 \gamma / 3>\gamma
$$

if $C>5 \gamma$.

For every $p$ with $\tilde{L}_{3}(p)-\tilde{L}_{2}(p)=\gamma$, hence $\tilde{L}_{2}(p)-\tilde{L}_{1}(p)>\gamma$, there is a smallest $a$ and a largest $b$ with $a \leq p \leq b$ such that

$$
\tilde{L}_{3}(a)-\tilde{L}_{2}(a)=\tilde{L}_{3}(b)-\tilde{L}_{2}(b)=\gamma
$$

and $\tilde{L}_{2}(q)-\tilde{L}_{1}(q)>\gamma$ for $a \leq q \leq b$. Such an interval $[a, b]$ will be called a top interval. It is not required that $\tilde{L}_{3}(q)-\tilde{L}_{2}(q) \leq \gamma$ in this interval. Also, it may happen that $a=p=b$, so that the interval consists of a single number.

For every $p^{*}$ with $\tilde{L}_{2}\left(p^{*}\right)-\tilde{L}_{1}\left(p^{*}\right)=\gamma$ there is a smallest $a^{*}$ and a largest $b^{*}$ with $a^{*} \leq p^{*} \leq b^{*}$ such that

$$
\tilde{L}_{2}\left(a^{*}\right)-\tilde{L}_{1}\left(a^{*}\right)=\tilde{L}_{2}\left(b^{*}\right)-\tilde{L}_{1}\left(b^{*}\right)=\gamma
$$

and $\tilde{L}_{3}(q)-\tilde{L}_{2}(q)>\gamma$ for $a^{*} \leq q \leq b^{*}$. Such an interval $\left[a^{*}, b^{*}\right]$ will be called a bottom interval. By (6.2), a top interval has distance greater than $\gamma$ from a bottom interval.

For intervals $I=[r, s]$ and $I^{\prime}=\left[r^{\prime}, s^{\prime}\right]$ we write $I<I^{\prime}$ if $s<r^{\prime}$. We may thus arrange all the top and bottom intervals into a sequence

$$
I_{1}<I_{2}<I_{3}<\cdots \text {. }
$$

There cannot be two adjacent top intervals in this sequence: for if $[a, b]<$ $\left[a^{\prime}, b^{\prime}\right]$ were two such intervals, then $\tilde{L}_{2}(q)-\tilde{L}_{1}(q)>\gamma$ for $a \leq q \leq b^{\prime}$, and $b$ would no longer be the largest number as required in the definition of top intervals. Similarly, there cannot be two adjacent bottom intervals. Hence after a change of notation, our sequence becomes

$$
I_{1}<I_{1}^{*}<I_{2}<I_{2}^{*}<\cdots,
$$

where, say, each $I_{j}$ is a top interval and each $I_{j}^{*}$ a bottom interval. If $I_{j}=$ $\left[a_{j}, b_{j}\right]$ and $I_{j}^{*}=\left[a_{j}^{*}, b_{j}^{*}\right]$ then

$$
\cdots<a_{j-1}^{*} \leq b_{j-1}^{*}<a_{j} \leq b_{j}<a_{j}^{*} \leq b_{j}^{*}<a_{j+1} \leq b_{j+1}<\cdots .
$$


In $\left[b_{j-1}^{*}, a_{j}^{*}\right]$ we have $\tilde{L}_{2}(q)-\tilde{L}_{1}(q)>\gamma$ and hence, by Lemma $5.1(\mathrm{vi})$, the function $\tilde{L}_{1}$ has no local maximum. There will be some $p_{j}^{*}$ in $\left[b_{j-1}^{*}, a_{j}^{*}\right]$ such that $\tilde{L}_{1}$ is decreasing for $b_{j-1}^{*} \leq q \leq p_{j}^{*}$ and increasing for $p_{j}^{*} \leq q \leq a_{j}^{*}$. The cases $p_{j}^{*}=b_{j-1}^{*}$ and $p_{j}^{*}=a_{j}^{*}$ are not ruled out. Also, there will be a $p_{j}$ in $\left[b_{j}, a_{j+1}\right]$ such that $\tilde{L}_{3}$ is increasing for $b_{j} \leq q \leq p_{j}$ and decreasing for $p_{j} \leq q \leq a_{j+1}$.

Let now $j$ be fixed and assume $j$ is large, so that $q \in I_{j}$ is large. We claim that we cannot have both $p_{j}^{*}, p_{j}$ larger than $b_{j}$. For if this were so, set $p=\min \left\{p_{j}^{*}, p_{j}\right\}$ and observe that $\tilde{L}_{3}$ is increasing in $\left[b_{j}, p\right] \subset\left[b_{j}, p_{j}\right]$ with slope $1 / 2$ and $\tilde{L}_{1}$ is decreasing in $\left[b_{j}, p\right] \subset\left[b_{j-1}^{*}, p_{j}\right]$ with slope -1 , so that $\tilde{L}_{2}$ is increasing with slope $1 / 2$. Hence

$$
\tilde{L}_{3}(q)-\tilde{L}_{2}(q)=\tilde{L}_{3}\left(b_{j}\right)-\tilde{L}_{2}\left(b_{j}\right)=\gamma
$$

in $\left[b_{j}, p\right]$, and there is no $q$ in this interval with $\tilde{L}_{2}(q)-\tilde{L}_{1}(q) \leq \gamma$. This contradicts the maximality property of the right endpoint $b_{j}$ of $I_{j}=\left[a_{j}, b_{j}\right]$. A similar reasoning shows that we cannot have both $p_{j}^{*}, p_{j}$ smaller than $a_{j}^{*}$. This yields the following possibilities. If $p_{j}^{*} \leq b_{j}$, then $p_{j} \geq a_{j}^{*}$, and conversely, $p_{j} \geq a_{j}^{*}$ implies $p_{j}^{*} \leq b_{j}$. In short,

$$
p_{j}^{*} \leq b_{j} \quad \text { and } \quad p_{j} \geq a_{j}^{*} .
$$

The second conceivable possibility is when $p_{j}^{*}>b_{j}$ and $p_{j}<a_{j}^{*}$, but then, by the above remarks and the fact that $p_{j}^{*} \leq a_{j}^{*}, p_{j} \geq b_{j}$, we have

$$
p_{j}=b_{j} \quad \text { and } \quad p_{j}^{*}=a_{j}^{*} \text {. }
$$

In fact, this cannot occur if $C$ is chosen sufficiently large. For if $p_{j}=b_{j}$ and $p_{j}^{*}=a_{j}^{*}$, both $\tilde{L}_{1}, \tilde{L}_{3}$ have slope -1 in $\left[b_{j}, a_{j}^{*}\right]$ so that $a_{j}^{*}-b_{j}<3 c$ by Lemma 5.1(v). Further, $\tilde{L}_{1}, \tilde{L}_{2}$ are close to each other at $q=a_{j}^{*}$ and $\tilde{L}_{2}, \tilde{L}_{3}$ are close at $q=b_{j}$, so that $\tilde{L}_{3}-\tilde{L}_{1}$ is small in $\left[b_{j}, a_{j}^{*}\right]$, i.e. $\tilde{L}_{3}-\tilde{L}_{1} \ll c$, which for large $C$ contradicts (6.1).

Let us now assume $p_{j}^{*} \leq b_{j}$ and $p_{j} \geq a_{j}^{*}$, so that we have $b_{j}^{*} \leq p_{j}^{*} \leq b_{j}$ $<a_{j}^{*}$. Then $\tilde{L}_{1}$ and $\tilde{L}_{3}$ are both increasing in $\left[b_{j}, a_{j}^{*}\right]$ with slope $1 / 2$, hence $\tilde{L}_{2}$ is decreasing with slope -1 . In fact, we may suppose that $p_{j}^{*}<a_{j}$, hence

$$
b_{j-1}^{*} \leq p_{j}^{*}<a_{j} \leq b_{j}<a_{j}^{*} .
$$

For otherwise $p_{j}^{*} \geq a_{j}$ and $\tilde{L}_{1}$ has slope -1 in $\left[b_{j-1}^{*}, p_{j}^{*}\right]$, hence in $\left[b_{j-1}^{*}, a_{j}\right]$. By Lemma 5.1(viii),

$$
\left|\tilde{L}_{3}\left(b_{j-1}^{*}\right)-L_{3}(q)-\left(\tilde{L}_{2}\left(b_{j-1}^{*}\right)-\tilde{L}_{2}(q)\right)\right|<8 c
$$

for $q$ in this interval, and since $\tilde{L}_{3}\left(a_{j}\right)-\tilde{L}_{2}\left(a_{j}\right)=\gamma$, we deduce that $\left|\tilde{L}_{3}\left(b_{j-1}^{*}\right)-\tilde{L}_{2}\left(b_{j-1}^{*}\right)\right|<8 c+\gamma=12 c$. Moreover, $\tilde{L}_{2}\left(b_{j-1}^{*}\right)-\tilde{L}_{1}\left(b_{j-1}^{*}\right)=\gamma$, 
so that

$$
\tilde{L}_{3}\left(b_{j-1}^{*}\right)-\tilde{L}_{1}\left(b_{j-1}^{*}\right)<12 c+\gamma=16 c .
$$

But this contradicts (6.1) provided $C$ is chosen large enough, i.e. $C>16 c$. Similarly, by a dual argument, we will also have

$$
b_{j}<a_{j}^{*} \leq b_{j}^{*}<p_{j} \leq a_{j+1} .
$$

The following figure illustrates a possible behavior of $\tilde{L}_{1}, \tilde{L}_{2}, \tilde{L}_{3}$ for $p_{j}^{*} \leq$ $q \leq a_{j+1}$

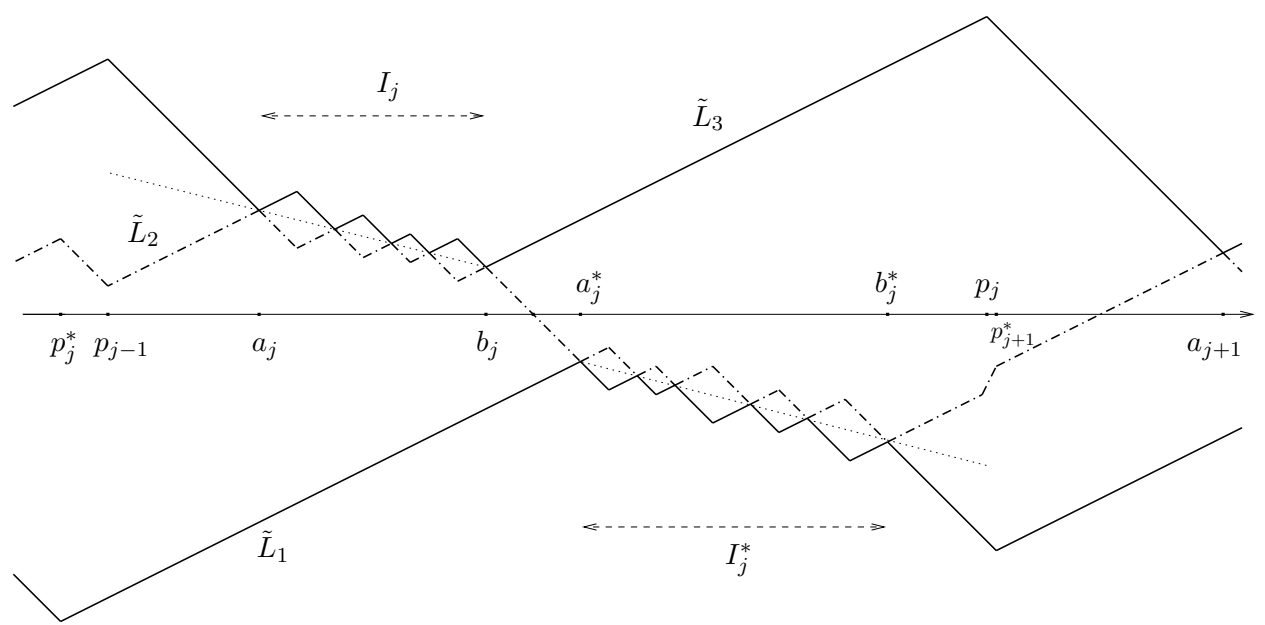

In $\left[a_{j}, b_{j}\right]$ the function $\tilde{L}_{1}$ has slope $1 / 2$, so that by Lemma 5.1 (vii), $\tilde{L}_{2}, \tilde{L}_{3}$ will zigzag around a line with slope $-1 / 4$ indicated by dots in the figure. The graphs of the functions are different near $p_{j}^{*}, p_{j-1}$ depending on whether $p_{j}^{*}<p_{j-1}$ (as shown in the figure) or $p_{j}^{*} \geq p_{j-1}$ (which in the figure holds for $j+1$ in place of $j$, i.e. $\left.p_{j+1}^{*} \geq p_{j}\right)$. Note that $p_{j}^{*} \geq p_{j-1}$ yields $p_{j}^{*}-p_{j-1}<3 c$ by Lemma $5.1(\mathrm{v})$.

Remarks. (a) In the notation of Section 4, suppose the local minima of $L_{1}=\tilde{L}_{1}$ in $\left[p_{j}^{*}, p_{j+1}^{*}\right]$ are assumed at $p_{j}^{*}=q_{k}<q_{k+1}<\cdots<q_{l}=p_{j+1}^{*}$. Then the points $\mathbf{x}_{k}, \mathbf{x}_{k+1}, \ldots, \mathbf{x}_{l}$ span a 2-dimensional space. For otherwise $l \geq$ $k+2$, and there is an $r, k<r<l$, with $\mathbf{x}_{k}, \mathbf{x}_{r}, \mathbf{x}_{l}$ linearly independent. If $\bar{q}$ is the $q$-coordinate of the point of intersection of the graphs of $L_{\mathbf{x}_{k}}, L_{\mathbf{x}_{l}}$, then it is clear from our figure that both $q_{r}, \bar{q}$ lie in the interior of $I_{j}^{*}=\left[a_{j}^{*}, b_{j}^{*}\right]$; in particular, $a_{j}^{*}<b_{j}^{*}$. Moreover, $L_{\mathbf{x}_{k}}\left(q_{k}\right)=L_{1}\left(q_{k}\right)=\tilde{L}_{1}\left(q_{k}\right)$, and both $L_{\mathbf{x}_{k}}$ and $\tilde{L}_{1}$ have slope $1 / 2$ in $q_{k} \leq q \leq a_{j}^{*}$, so that $L_{\mathbf{x}_{k}}\left(a_{j}^{*}\right)=\tilde{L}_{1}\left(a_{j}^{*}\right)<\tilde{L}_{3}\left(a_{j}^{*}\right)-\gamma$. Both $L_{\mathbf{x}_{k}}$ and $\tilde{L}_{3}$ have slope $1 / 2$ in $a_{j}^{*} \leq q \leq \bar{q}$, so that $L_{\mathbf{x}_{k}}(\bar{q})<\tilde{L}_{3}(\bar{q})-\gamma$ $<L_{3}(\bar{q})$. But by the independence of $\mathbf{x}_{k}, \mathbf{x}_{r}, \mathbf{x}_{l}$, and since $L_{\mathbf{x}_{k}}(\bar{q})=L_{\mathbf{x}_{l}}(\bar{q})$ $\geq L_{\mathbf{x}_{r}}(\bar{q})$, we have $L_{3}(\bar{q}) \leq L_{\mathbf{x}_{k}}(\bar{q})$, a contradiction. 
(b) When $a_{j}=b_{j}$ or $a_{j}^{*}=b_{j}^{*}$, which we believe to be a relatively common event, the figure is much simplified. (In fact, keeping the notations from (a) we have $a_{j}^{*}=b_{j}^{*}$ if $\mathbf{x}_{k}, \mathbf{x}_{k+1}, \mathbf{x}_{k+2}$ are linearly independent.)

(c) We believe that our description of the functions $\tilde{L}_{1}(q), \tilde{L}_{2}(q), \tilde{L}_{3}(q)$, hence implicitly of $\lambda_{1}(Q), \lambda_{2}(Q), \lambda_{3}(Q)$, is close to "best possible". That is, given functions as described in the figure, there are numbers $\xi_{1}, \xi_{2}$ for which the resulting $\tilde{L}_{1}, \tilde{L}_{2}, \tilde{L}_{3}$ are close to the given functions.

7. Proof of Theorem 1.5. We introduce the functions $\tilde{\chi}_{i}(q):=\tilde{L}_{i}(q) / q$ for $i=1,2,3$. Then

$$
\tilde{\chi}_{i}(q)-\psi_{i}\left(e^{q}\right)=\frac{\tilde{L}_{i}(q)}{q}-\frac{L_{i}(q)}{q} \ll \frac{1}{q}
$$

so that we have

$$
\bar{\psi}_{i}=\limsup \tilde{\chi}_{i}(q) \quad \text { and } \quad \underline{\psi}_{i}=\liminf \tilde{\chi}_{i}(q)
$$

by (4.10). Moreover, by the case $n=3$ of (4.9) we have the estimates

$$
\tilde{\chi}_{i}(q) \geq-1+\frac{g_{1}\left(e^{q}\right)}{q} \text { and } \tilde{\chi}_{i}(q) \leq \frac{1}{2}-\frac{g_{3}\left(e^{q}\right)}{q},
$$

hence for large $q$,

$$
-1<\tilde{\chi}_{i}(q)<1 / 2 \quad \text { for } i=1,2,3 \text {. }
$$

LEMMA 7.1.

$$
\tilde{L}_{1}\left(a_{j}^{*}\right)+\tilde{L}_{3}\left(b_{j}\right)=O(1), \quad \tilde{\chi}_{1}\left(a_{j}^{*}\right)+\tilde{\chi}_{3}\left(b_{j}\right)=-2 \tilde{\chi}_{1}\left(a_{j}^{*}\right) \tilde{\chi}_{3}\left(b_{j}\right)+O\left(1 / b_{j}\right),
$$

where the implied constants depend only on $c$, hence are absolute.

Proof. We will drop the subscript $j$. We have $\tilde{L}_{2}\left(a^{*}\right)-\tilde{L}_{1}\left(a^{*}\right)=O(1)$, hence $2 \tilde{L}_{1}\left(a^{*}\right)+\tilde{L}_{3}\left(a^{*}\right)=O(1)$. Similarly, $2 \tilde{L}_{3}(b)+\tilde{L}_{1}(b)=O(1)$. Both $\tilde{L}_{1}, \tilde{L}_{3}$ have slope $1 / 2$ in $\left[b, a^{*}\right]$, so that

$$
\tilde{L}_{3}\left(a^{*}\right)-\tilde{L}_{3}(b)=\frac{1}{2}\left(a^{*}-b\right)=\tilde{L}_{1}\left(a^{*}\right)-\tilde{L}_{1}(b),
$$

which yields

$$
\tilde{L}_{1}(b)+\tilde{L}_{3}\left(a^{*}\right)=\tilde{L}_{3}(b)+\tilde{L}_{1}\left(a^{*}\right) .
$$

Therefore

$$
\tilde{L}_{1}\left(a^{*}\right)+\tilde{L}_{3}(b)=\frac{1}{3}\left(2 \tilde{L}_{1}\left(a^{*}\right)+\tilde{L}_{3}\left(a^{*}\right)+2 \tilde{L}_{3}(b)+\tilde{L}_{1}(b)\right)=O(1) .
$$

Also,

$$
\tilde{L}_{3}\left(a^{*}\right)=-\tilde{L}_{1}\left(a^{*}\right)-\tilde{L}_{2}\left(a^{*}\right)=-2 \tilde{L}_{1}\left(a^{*}\right)+O(1)=2 \tilde{L}_{3}(b)+O(1),
$$

so that

$$
a^{*}-b=2\left(\tilde{L}_{3}\left(a^{*}\right)-\tilde{L}_{3}(b)\right)=2 \tilde{L}_{3}(b)+O(1) .
$$


Therefore

$$
\begin{aligned}
\tilde{\chi}_{1}\left(a^{*}\right)+\tilde{\chi}_{3}(b) & =\frac{1}{a^{*} b}\left(b \tilde{L}_{1}\left(a^{*}\right)+a^{*} \tilde{L}_{3}(b)\right) \\
& =\frac{1}{a^{*} b}\left(b \tilde{L}_{1}\left(a^{*}\right)-a^{*} \tilde{L}_{1}\left(a^{*}\right)\right)+O(1 / b) \\
& =\frac{1}{a^{*} b}\left(\left(b-a^{*}\right) \tilde{L}_{1}\left(a^{*}\right)\right)+O(1 / b) \\
& =-\frac{2}{a^{*} b} \tilde{L}_{1}\left(a^{*}\right) \tilde{L}_{3}(b)+O(1 / b) \\
& =-2 \tilde{\chi}_{1}\left(a^{*}\right) \tilde{\chi}_{3}(b)+O(1 / b) .
\end{aligned}
$$

LEMMA 7.2.

(i) If some $\tilde{L}_{i}$ is increasing (resp. decreasing) in an interval with large endpoints, then also $\tilde{\chi}_{i}$ is increasing (resp. decreasing) in that interval.

(ii) For $q \in I_{j}^{*}=\left[a_{j}^{*}, b_{j}^{*}\right]$ with large $j$ we have $\tilde{\chi}_{1}(q) \leq \tilde{\chi}_{1}\left(a_{j}^{*}\right)+O\left(1 / a_{j}^{*}\right)$.

(iii) For $q \in I_{j}=\left[a_{j}, b_{j}\right]$ with large $j$ we have $\tilde{\chi}_{3}(q) \geq \tilde{\chi}_{3}\left(b_{j}\right)-O\left(1 / b_{j}\right)$.

Proof. (i) Suppose $q>q^{\prime}$ are in the interval. If $\tilde{L}_{i}$ is increasing, then $\tilde{L}_{i}(q)-\tilde{L}_{i}\left(q^{\prime}\right) \geq \frac{1}{2}\left(q-q^{\prime}\right)$, yielding

$\tilde{\chi}_{i}(q) \geq \tilde{\chi}_{i}\left(q^{\prime}\right) q^{\prime} / q+\frac{1}{2}\left(1-q^{\prime} / q\right)=\tilde{\chi}_{i}\left(q^{\prime}\right)+\left(\frac{1}{2}-\tilde{\chi}_{i}\left(q^{\prime}\right)\right)\left(1-q^{\prime} / q\right)>\tilde{\chi}_{i}\left(q^{\prime}\right)$

by (7.3). If $\tilde{L}_{i}$ is decreasing, then $\tilde{L}_{i}(q)-\tilde{L}_{i}\left(q^{\prime}\right)=-\left(q-q^{\prime}\right)$, so that

$$
\tilde{\chi}_{i}(q)=\tilde{\chi}_{i}\left(q^{\prime}\right) q^{\prime} / q-\left(1-q^{\prime} / q\right)=\tilde{\chi}_{i}\left(q^{\prime}\right)-\left(1+\tilde{\chi}_{i}\left(q^{\prime}\right)\right)\left(1-q^{\prime} / q\right)<\tilde{\chi}_{i}\left(q^{\prime}\right)
$$
by (7.3).

(ii) We have

$$
\tilde{L}_{1}\left(a_{j}^{*}\right) \geq \frac{1}{2}\left(\tilde{L}_{1}\left(a_{j}^{*}\right)+\tilde{L}_{2}\left(a_{j}^{*}\right)\right)-O(1)=-\frac{1}{2} \tilde{L}_{3}\left(a_{j}^{*}\right)-O(1),
$$

so that for large $j$,

$$
\tilde{\chi}_{1}\left(a_{j}^{*}\right) \geq-\frac{1}{2} \tilde{\chi}_{3}\left(a_{j}^{*}\right)-O\left(1 / a_{j}^{*}\right)
$$

and hence (7.2) yields

$$
\tilde{\chi}_{1}\left(a_{j}^{*}\right)>-\frac{1}{4}+\frac{1}{2} g_{3}\left(e^{a_{j}^{*}}\right) / a_{j}^{*}-O\left(1 / a_{j}^{*}\right)>-\frac{1}{4} .
$$

Moreover

$$
\begin{aligned}
\tilde{L}_{1}(q) & \leq \frac{1}{2}\left(\tilde{L}_{1}(q)+\tilde{L}_{2}(q)\right)+O(1) \\
& =\frac{1}{2}\left(\tilde{L}_{1}\left(a_{j}^{*}\right)+\tilde{L}_{2}\left(a_{j}^{*}\right)\right)-\frac{1}{4}\left(q-a_{j}^{*}\right)+O(1) \\
& =\tilde{L}_{1}\left(a_{j}^{*}\right)-\frac{1}{4}\left(q-a_{j}^{*}\right)+O(1),
\end{aligned}
$$


hence by (7.4),

$$
\begin{aligned}
\tilde{\chi}_{1}(q) & \leq \tilde{\chi}_{1}\left(a_{j}^{*}\right) a_{j}^{*} / q-\frac{1}{4}\left(1-a_{j}^{*} / q\right)+O(1 / q) \\
& =\tilde{\chi}_{1}\left(a_{j}^{*}\right)-\left(\frac{1}{4}+\tilde{\chi}_{1}\left(a_{j}^{*}\right)\right)\left(1-a_{j}^{*} / q\right)+O(1 / q) \leq \tilde{\chi}_{1}\left(a_{j}^{*}\right)+O\left(1 / a_{j}^{*}\right) .
\end{aligned}
$$

(iii) is proved in a dual way.

Set $\mathcal{I}_{j}:=\left[p_{j-1}, p_{j}\right], \mathcal{I}_{j}^{*}:=\left[p_{j}^{*}, p_{j+1}^{*}\right]$ and put

$$
\rho_{j}:=\max _{q \in \mathcal{I}_{j}^{*}} \tilde{\chi}_{1}(q), \quad \sigma_{j}:=\min _{q \in \mathcal{I}_{j}} \tilde{\chi}_{3}(q) .
$$

LEMMA 7.3.

$$
\begin{aligned}
& \text { (i) } \tilde{\chi}_{1}\left(a_{j}^{*}\right) \leq \rho_{j}<\tilde{\chi}_{1}\left(a_{j}^{*}\right)+O\left(1 / a_{j}^{*}\right), \\
& \text { (ii) } \tilde{\chi}_{3}\left(b_{j}\right) \geq \sigma_{j}>\tilde{\chi}_{3}\left(b_{j}\right)-O\left(1 / b_{j}\right) .
\end{aligned}
$$

Proof. Since $\tilde{L}_{1}$ is increasing in $\left[p_{j-1}, a_{j}^{*}\right]$ and decreasing in $\left[b_{j}^{*}, p_{j}\right]$, we see from Lemma $7.2(\mathrm{i})$ that $\rho_{j}=\tilde{\chi}_{1}(q)$ with $q \in\left[a_{j}^{*}, b_{j}^{*}\right]=I_{j}^{*}$. But by Lemma $7.2(\mathrm{ii})$, in fact $\rho_{j}<\tilde{\chi}_{1}\left(a_{j}^{*}\right)+O\left(1 / a_{j}^{*}\right)$. Now (i) follows, and (ii) is proved in a dual fashion.

Proof of Theorem 1.5. By combining Lemmas 7.1 and 7.3 we obtain

$$
\rho_{j}+\sigma_{j}+2 \rho_{j} \sigma_{j}=O\left(1 / \min \left\{b_{j}, a_{j}^{*}\right\}\right)=O\left(1 / b_{j}\right) .
$$

For large $j$ the inequalities $-1 / 3<\rho_{j} \leq 0$ hold by (7.4) and Lemma 7.3(i), so that $1+2 \rho_{j}>1 / 3$ and hence

$$
\sigma_{j}=-\frac{\rho_{j}}{1+2 \rho_{j}}+O\left(1 / b_{j}\right)
$$

All large reals lie in the union of the intervals $\mathcal{I}_{2}, \mathcal{I}_{3}, \ldots$ as well as of the intervals $\mathcal{I}_{1}^{*}, \mathcal{I}_{2}^{*}, \ldots$, and as the function $\rho \mapsto \rho /(1+2 \rho)$ for $\rho>-1 / 2$ is increasing, we conclude

$$
\underline{\psi}_{3}=\liminf \sigma_{j}=\liminf \left(-\frac{\rho_{j}}{1+2 \rho_{j}}\right)=-\frac{\lim \sup \rho_{j}}{1+2 \lim \sup \rho_{j}}=-\frac{\bar{\psi}_{1}}{1+2 \bar{\psi}_{1}},
$$

and this is precisely (1.11).

8. Proof of Theorem 1.6. Let $\mathcal{K}_{j}$ be the interval $\left[b_{j-1}, b_{j}\right]$ and $\mathcal{K}_{j}^{*}$ be the interval $\left[a_{j}^{*}, a_{j+1}^{*}\right]$. Further, let $m_{j}, m_{j}^{*}$ be numbers in $\mathcal{K}_{j}, \mathcal{K}_{j}^{*}$ respectively with

$$
\psi_{3}\left(m_{j}\right)=\max _{q \in \mathcal{K}_{j}} \psi_{3}(q) \quad \text { and } \quad \psi_{1}\left(m_{j}^{*}\right)=\min _{q \in \mathcal{K}_{j}^{*}} \psi_{1}(q) .
$$

LEMMA 8.1.

$$
\begin{aligned}
& 2 \tilde{\chi}_{1}\left(m_{j}\right)+\tilde{\chi}_{3}\left(m_{j}\right) \leq-\tilde{\chi}_{3}\left(b_{j}\right)\left(3+2 \tilde{\chi}_{1}\left(m_{j}\right)+4 \tilde{\chi}_{3}\left(m_{j}\right)\right)+O\left(1 / m_{j}\right) \\
& 2 \tilde{\chi}_{3}\left(m_{j}^{*}\right)+\tilde{\chi}_{1}\left(m_{j}^{*}\right) \geq-\tilde{\chi}_{1}\left(a_{j}^{*}\right)\left(3+2 \tilde{\chi}_{3}\left(m_{j}^{*}\right)+4 \tilde{\chi}_{1}\left(m_{j}^{*}\right)\right)+O\left(1 / m_{j}^{*}\right)
\end{aligned}
$$


Proof. Since $\tilde{\chi}_{3}$ increases in $\left[b_{j-1}, p_{j-1}\right]$ by Lemma 7.2 , we have $m_{j} \in$ $\left[p_{j-1}, b_{j}\right]$. Now $L_{1}$ increases with slope $1 / 2$ in $\left[p_{j}^{*}, p_{j-1}\right]$. Either $p_{j}^{*}<p_{j-1}$, or $p_{j}^{*} \geq p_{j-1}$ and $p_{j}^{*}-p_{j-1}=O(1)$. In either case, since $m_{j} \in\left[p_{j-1}, b_{j}\right]$, we have

$$
\tilde{L}_{1}\left(m_{j}\right)=\tilde{L}_{1}\left(b_{j}\right)-\frac{1}{2}\left(b_{j}-m_{j}\right)+O(1) .
$$

Since $m_{j} \leq b_{j}$ and $\tilde{L}_{3}\left(b_{j}\right)$ has slopes among $-1,1 / 2$,

$$
\tilde{L}_{3}\left(m_{j}\right) \leq \tilde{L}_{3}\left(b_{j}\right)+\left(b_{j}-m_{j}\right)
$$

Therefore

$$
\begin{aligned}
2 \tilde{L}_{1}\left(m_{j}\right)+\tilde{L}_{3}\left(m_{j}\right) & \leq 2 \tilde{L}_{1}\left(b_{j}\right)+\tilde{L}_{3}\left(b_{j}\right)+O(1)=-2 \tilde{L}_{2}\left(b_{j}\right)-\tilde{L}_{3}\left(b_{j}\right)+O(1) \\
& =-3 \tilde{L}_{3}\left(b_{j}\right)+O(1) .
\end{aligned}
$$

Dividing by $m_{j}$ we obtain

$$
2 \tilde{\chi}_{1}\left(m_{j}\right)+\tilde{\chi}_{3}\left(m_{j}\right) \leq-3 \tilde{\chi}_{3}\left(b_{j}\right) b_{j} / m_{j}+O\left(1 / m_{j}\right) .
$$

On the other hand,

$$
\begin{aligned}
2 \tilde{L}_{1}\left(m_{j}\right)+4 \tilde{L}_{3}\left(m_{j}\right) & \leq 2 \tilde{L}_{1}\left(b_{j}\right)+4 \tilde{L}_{3}\left(b_{j}\right)+3\left(b_{j}-m_{j}\right)+O(1) \\
& =-2 \tilde{L}_{2}\left(b_{j}\right)+2 \tilde{L}_{3}\left(b_{j}\right)+3\left(b_{j}-m_{j}\right)+O(1) \\
& =3\left(b_{j}-m_{j}\right)+O(1) .
\end{aligned}
$$

After division by $m_{j}$ and addition of 3 we get

$$
3+2 \tilde{\chi}_{1}\left(m_{j}\right)+4 \tilde{\chi}_{3}\left(m_{j}\right) \leq 3 b_{j} / m_{j}+O\left(1 / m_{j}\right) .
$$

This, in conjunction with (8.3), gives (8.1).

(8.2) is proved in a dual fashion: there is some symmetry of the graphs of $\tilde{L}_{1}, \tilde{L}_{2}, \tilde{L}_{3}$ about the point $\left(\frac{1}{2}\left(b_{j}+a_{j}^{*}\right), 0\right)$. This is not a strict geometric symmetry, but our arguments remain valid if $p_{j-1}, a_{j}, b_{j}, a_{j}^{*}, b_{j}^{*}, p_{j+1}^{*}$ are respectively replaced by $p_{j+1}^{*}, b_{j}^{*}, a_{j}^{*}, b_{j}, a_{j}, p_{j-1}$, if $\tilde{L}_{1}, \tilde{L}_{2}, \tilde{L}_{3}$ are respectively replaced by $\tilde{L}_{3}, \tilde{L}_{2}, \tilde{L}_{1}$, and inequalities are reversed. Thus for instance $\tilde{\chi}_{3}\left(b_{j}\right)=\tilde{L}_{3}\left(b_{j}\right) / b_{j}$ is replaced by $\tilde{\chi}_{1}\left(a_{j}^{*}\right)=\tilde{L}_{1}\left(a_{j}^{*}\right) / a_{j}^{*}$. by

We now have $m_{j}^{*} \in\left[a_{j}^{*}, p_{j+1}^{*}\right]$, and (8.3), (8.4) are respectively replaced

$$
\begin{aligned}
2 \tilde{\chi}_{3}\left(m_{j}^{*}\right)+\tilde{\chi}_{1}\left(m_{j}^{*}\right) & \geq-3 \tilde{\chi}_{1}\left(a_{j}^{*}\right) a_{j}^{*} / m_{j}^{*}+O\left(1 / m_{j}^{*}\right), \\
3+2 \tilde{\chi}_{3}\left(m_{j}^{*}\right)+4 \tilde{\chi}_{1}\left(m_{j}^{*}\right) & \geq 3 a_{j}^{*} / m_{j}^{*}+O\left(1 / m_{j}^{*}\right) .
\end{aligned}
$$

Thus (8.2) follows.

Proof of Theorem 1.6. For given $\epsilon>0$ we have $\tilde{\chi}_{3}\left(b_{j}\right)>\underline{\psi}_{3}-\epsilon$, and (8.1) yields

$$
2 \tilde{\chi}_{1}\left(m_{j}\right)+\tilde{\chi}_{3}\left(m_{j}\right) \leq-\left(\underline{\psi}_{3}-\epsilon\right)\left(3+2 \tilde{\chi}_{1}\left(m_{j}\right)+4 \tilde{\chi}_{3}\left(m_{j}\right)\right)+O\left(1 / m_{j}\right),
$$

hence

$$
\tilde{\chi}_{3}\left(m_{j}\right)\left(1+4 \underline{\psi}_{3}\right)<-3 \underline{\psi}_{3}-\left(2+2 \underline{\psi}_{3}\right) \tilde{\chi}_{1}\left(m_{j}\right)+O(\epsilon)
$$


when $j$ is large. Moreover, $\tilde{\chi}_{1}\left(m_{j}\right)>\underline{\psi}_{1}-\epsilon$, so that

$$
\tilde{\chi}_{3}\left(m_{j}\right)\left(1+4 \underline{\psi}_{3}\right)<-3 \underline{\psi}_{3}-\left(2+2 \underline{\psi}_{3}\right) \underline{\psi}_{1}+O(\epsilon) .
$$

Since by definition of $m_{j}$,

$$
\bar{\psi}_{3}=\limsup _{j \rightarrow \infty} \tilde{\chi}_{3}\left(m_{j}\right)
$$

we obtain

$$
\bar{\psi}_{3}\left(1+4 \underline{\psi}_{3}\right) \leq-3 \underline{\psi}_{3}-\left(2+2 \underline{\psi}_{3}\right) \underline{\psi}_{1},
$$

hence (1.12). The relation (1.13) is established in a dual way.

\section{Some consequences}

Corollary 9.1. Again in the context of Dirichlet's Theorem on simultaneous approximation with $n=3$,

$$
\bar{\psi}_{3} \geq \underline{\psi}_{3}\left(3+2 \underline{\psi}_{1}\right) \quad \text { and } \quad \underline{\psi}_{1} \leq \bar{\psi}_{1}\left(3+2 \bar{\psi}_{3}\right) .
$$

Proof. (1.12) and (1.15) can be rewritten as $3 \underline{\psi}_{3}+2 \underline{\psi}_{1}+\bar{\psi}_{3} \leq \underline{\psi}_{3}\left(-2 \underline{\psi}_{1}-4 \bar{\psi}_{3}\right), \quad 3 \underline{\psi}_{3}-\underline{\psi}_{1}-2 \bar{\psi}_{3} \leq \underline{\psi}_{3}\left(-2 \underline{\psi}_{1}+2 \bar{\psi}_{3}\right)$. Adding the first of these inequalities to twice the second, we obtain

$$
9 \underline{\psi}_{3}-3 \bar{\psi}_{3} \leq-6 \underline{\psi}_{1} \underline{\psi}_{3},
$$

which gives the first relation of (9.1). We noted that $(1.12), \ldots,(1.15)$ remain valid if $\underline{\psi}_{1}, \bar{\psi}_{3}$ as well as $\bar{\psi}_{1}, \underline{\psi}_{3}$ are interchanged and inequalities are reversed. This also holds for the consequences of $(1.12), \ldots,(1.15)$, and hence the first relation of (9.1) implies the second.

Corollary 9.2. If $\underline{\psi}_{1}=\bar{\psi}_{1}$ or $\underline{\psi}_{2}=\bar{\psi}_{2}$, then

$$
\underline{\psi}_{i}=\bar{\psi}_{i} \quad \text { for } i=1,2,3 \text {. }
$$

When $\underline{\psi}_{3}=\bar{\psi}_{3}$, then either (9.2) holds or

$$
\underline{\psi}_{1}=-1, \quad \bar{\psi}_{1}=\underline{\psi}_{2}=-1 / 4, \quad \bar{\psi}_{2}=\underline{\psi}_{3}=\bar{\psi}_{3}=1 / 2 .
$$

Proof. Assume $\underline{\psi}_{1}=\bar{\psi}_{1}$. Then we have $\underline{\psi}_{1}=\bar{\psi}_{1} \leq \bar{\psi}_{1}\left(3+2 \bar{\psi}_{3}\right)$ and hence either $\bar{\psi}_{1}=\underline{\psi}_{1}=0$ or $1=3+2 \bar{\psi}_{3} \geq 3$, which is impossible. Now by (1.5) we have $\underline{\psi}_{1} \leq \underline{\psi}_{2} \leq \bar{\psi}_{1}$, hence $\underline{\psi}_{2}=0$, and $\underline{\psi}_{1}+\underline{\psi}_{2}+\bar{\psi}_{3} \leq 0$ yields $\bar{\psi}_{2}=\underline{\psi}_{3}=\bar{\psi}_{3}=0$ so that (9.2) follows.

If $\underline{\psi}_{2}=\bar{\psi}_{2}$, we have $3 \underline{\psi}_{2}=3 \bar{\psi}_{2} \leq 2 \bar{\psi}_{2}+\underline{\psi}_{1}$, which implies $\bar{\psi}_{2} \leq \underline{\psi}_{1}$ and this is only possible if $\bar{\psi}_{2}=\underline{\psi}_{1}=0$. As before, this yields $\bar{\psi}_{3}=0$ as well, and again (9.2) follows.

Now let $\underline{\psi}_{3}=\bar{\psi}_{3}$. Then we have $\bar{\psi}_{3}=\underline{\psi}_{3} \geq \underline{\psi}_{3}\left(3+2 \underline{\psi}_{1}\right)$ and hence either $\bar{\psi}_{3}=\underline{\psi}_{3}=0$ or $1=3+2 \underline{\psi}_{1} \geq 3$, which is only possible if $\underline{\psi}_{1}=-1$. In the first case $\underline{\psi}_{3} \leq \bar{\psi}_{2} \leq \bar{\psi}_{3}$ (we used (1.5) again) implies $\bar{\psi}_{2}=0$, and 
$\underline{\psi}_{1}+\bar{\psi}_{2}+\bar{\psi}_{3} \geq 0$ yields $\underline{\psi}_{2}=\bar{\psi}_{1}=\underline{\psi}_{1}=0$ and hence $(9.2)$. In the second case $\underline{\psi}_{1}=-1$ and in view of $\underline{\psi}_{1}+\bar{\psi}_{2}+\bar{\psi}_{3} \geq 0$ we must have $\bar{\psi}_{2}=\underline{\psi}_{3}=\bar{\psi}_{3}=1 / 2$. Then $\bar{\psi}_{1}+\underline{\psi}_{3}+2 \bar{\psi}_{1} \underline{\psi}_{3}=0$ yields $\bar{\psi}_{1}=-1 / 4$, and $\underline{\psi}_{2} \leq \bar{\psi}_{1}$ gives $\underline{\psi}_{2}=-1 / 4$, which concludes the proof.

Acknowledgements. Research of L. Summerer supported by FWF grant P19994-N13.

\section{References}

[1] Y. Bugeaud and M. Laurent, On exponents of homogeneous and inhomogeneous Diophantine approximation, Moscow Math. J. 5 (2005), 747-766.

[2] —, 一, Exponents of Diophantine approximation, in: Diophantine Geometry, CRM Ser. 4, Ed. Norm., Pisa, 2007, 101-121.

[3] V. Jarník, Zum Khintchineschen "Übertragungssatz", Trav. Inst. Math. Tbilissi 3 (1938), 193-212.

[4] A. Y. Khintchine [A. Ya. Khinchin], Zur metrischen Theorie der diophantischen Approximationen, Math. Z. 24 (1926), 706-714.

[5] - Über eine Klasse linearer diophantischer Approximationen, Rend. Circ. Mat. Palermo 50 (1926), 170-195.

[6] M. Laurent, Exponents of Diophantine approximation in dimension two, Canad. J. Math. 61 (2009), 165-189.

[7] K. Mahler, The successive minima in the geometry of numbers and the distinction between algebraic and transcendental numbers, J. Number Theory 22 (1986), 147160 .

[8] H. Minkowski, Geometrie der Zahlen, Teubner, Leipzig, 1910.

[9] N. G. Moshchevitin, Best Diophantine approximations: the phenomenon of degenerate dimension, in: Surveys in Geometry and Number Theory: Report on Contemporary Russian Mathematics, London Math. Soc. Lecture Note Ser. 338, Cambridge Univ. Press, Cambridge, 2007, 158-182.

[10] - Proof of W. M. Schmidt's conjecture concerning successive minima of a lattice, preprint, arXiv:0804.0120.

[11] D. Roy, On two exponents of approximation related to a real number and its square, Canad. J. Math. 59 (2007), 211-224.

[12] W. M. Schmidt, Open problems in Diophantine approximation, in: Diophantine Approximations and Transcendental Numbers (Luminy, 1982), Progr. Math. 31, Birkhäuser, Boston, 1983, 271-287.

Department of Mathematics

University of Colorado

Boulder, CO 80309-0395, U.S.A.

E-mail: schmidt@euclid.colorado.edu
Faculty of Mathematics

University of Vienna

Strudlhofgasse 4

A-1090 Wien, Austria

E-mail: leonhard.summerer@univie.ac.at

Received on 6.1.2009

and in revised form on 19.3.2009 